TRANSACTIONS OF THE

AMERICAN MATHEMATICAL SOCIETY

Volume 281. Number 2. February 1984

\title{
ASYMPTOTIC DIRICHLET PROBLEMS FOR HARMONIC FUNCTIONS ON RIEMANNIAN MANIFOLDS
}

BY

\author{
HYEONG IN CHOI
}

\begin{abstract}
We define the asymptotic Dirichlet problem and give a sufficient condition for solving it. This proves an existence of nontrivial bounded harmonic functions on certain classes of noncompact complete Riemannian manifolds.
\end{abstract}

0. Introduction. In this paper we will prove the existence of nonconstant bounded harmonic functions on certain classes of noncompact Riemannian manifolds by defining and solving an asymptotically defined Dirichlet problem for harmonic functions. The motivation comes from the classical uniformization theorem of Riemann surfaces which says that a simply connected Riemann surface is biholomorphic to the Riemann sphere $S^{2}$, the complex plane $\mathbf{C}$, or the unit disk $D$. This is a geometric theorem, but its original proof due to Koebe relies heavily on function theory. The function-theoretic interpretation of this theorem is the following: Among the noncompact simply connected surfaces, $\mathbf{C}$ is characterized by the fact that it admits no nonconstant bounded harmonic functions, and $D$ by that it admits nonconstant bounded harmonic functions.

The geometric aspect of the uniformization theorem could be roughly stated as follows: Let $M$ be a simply connected Riemann surface equipped with a complete Riemannian metric with Gaussian curvature $K_{M}$, if $K_{M} \geqslant c>0$, then $M$ is biholomorphic to $S^{2}$; if $K_{M} \leqslant-c<0$, then $M$ is biholomorphic to $D$; if $K_{M}$ is "close" to zero, then $M$ is biholomorphic to $\mathbf{C}$. The following precise version is due to Greene and Wu [GW1, p. 120].

THEOREM 0.1. Let $M$ be a two-dimensional Riemannian manifold such that the exponential map at some point $p$ is a diffeomorphism. Let $r$ be the geodesic distance function from $p$.

(A) $M$ has the complex structure of the disk if its curvature is nonpositive and $\leqslant-(1+\varepsilon) / r^{2} \log r$ outside some compact set for some $\varepsilon>0$.

(B) $M$ has the complex structure of $\mathbf{C}$ if either one of the following conditions are satisfied:

(B1) Outside a disk of radius $r_{0}$, curvature $\geqslant-1 / r^{2} \log r$.

Received by the editors November 16, 1982.

1980 Mathematics Subject Classification. Primary 53C99. 
(B2) There exist nonnegative continuous functions $K, k:[0, \infty] \rightarrow[0, \infty]$ such that $K(r) \geqslant$ curvature at $q \geqslant-k(r)$ where $r=\operatorname{dist}(p, q)$ for all $q \in M$,

$$
\int_{0}^{\infty} s k(s)<\infty \text { and } \int_{0}^{\infty} s K(s) \leqslant 1
$$

The curious fact that the constants $1+\varepsilon$ in (A) and 1 in (B1) sharply distinguishes the two cases was first observed in Milnor [Mi] assuming $M$ is rotationally symmetric. Ahlfors $[\mathbf{A l}]$ and Yang [Ya] have earlier proved part (A) under stronger curvature assumptions.

As the above discussion suggests, the function theory and the geometry are closely tied together, and there have been a lot of activities concerning the generalization of this phenomenon to higher dimensions. Most outstanding are results by Greene and $\mathrm{Wu}$, and by Yau. The striking result of Greene and $\mathrm{Wu}$ [GW2] says that under an assumption on $M$ very similar to (B) of Theorem 0.1 and when $\operatorname{dim} M \geqslant 3 M$ is actually isometric to $\mathbf{R}^{n}$. Therefore, such $M$ cannot have a nonconstant bounded harmonic function by the Liouville theorem. In fact, Greene and Wu [GW1, p. 56] proved earlier that under the assumption of (B1) and regardless of the dimension of $M$, the exponential map is a quasi-isometry, therefore by Moser's Harnack inequality every positive harmonic function is constant. Yau's result [Yu] is that every positive harmonic function is constant on a complete Riemannian manifold with nonnegative Ricci curvature. This type of Liouville theorem has many generalizations to harmonic maps. The result of Hildebrandt, Jost and Widman [HJW] could be regarded as a generalization of Greene and Wu's result. Generalization of Yau's result was achieved by S. Y. Cheng [Ch] and the author [Ci1].

In contrast to the impressive success of the Liouville type nonexistence theorems, basically nothing is known about the existence theorem except for dimension 2 . Even in the two-dimensional case most known results use the uniformization theorem, which cannot be generalized to higher dimensions. There seems to be no effective direct geometric method known so far. In this paper we shall give a partial answer to the existence question which was conjectured by Greene and Wu [GW1, p. 3].

Philosophically, our problem is linked to the old conjecture of $\mathrm{Wu}[\mathbf{W}]$ which says roughly that a complete simply connected Kähler manifold with "sufficiently negative" curvature is biholomorphic to a bounded domain in $\mathbf{C}^{n}$, and our problem should be understood in this perspective.

This paper is divided into five sections. In $\S 1$ we shall give the definition of the Eberlein-O’Neill boundary, fix notations and state some well-known formulas. In $\$ 2$ we define the asymptotic Dirichlet problem, and by employing the classical Perron method we reduce the problem to the construction of barriers. The key concept is the barrier with angle which enables us to overcome the bad angular behavior. This is a technical change, but it turns out to play a very important role. In $\S 3$ under the assumption of rotational symmetry, our problem becomes essentially a one-dimensional one. It is studied mainly for the sake of motivation, but it also has some interesting features such as the use of the radial curvature only, the sharpness of the curvature decay, etc. We also give characterizations of rotationally symmetric manifolds. (See [Ci2] for more general results.) In $\S 4$, the general concept of barrier 
with angle is utilized and we reduce the problem to the question of convexity. We feel that this is one of our main contributions to this problem. In $\$ 5$, the asymptotic Dirichlet problem is generalized to nonsimply connected manifolds. Since the convexity question is easy to deal with on surfaces, we will mostly consider surfaces, even if a similar generalization is possible for higher dimensions. The asymptotic Dirichlet problem is posed on each "expanding" end. The idea of $\$ 4$ is fully exploited in this section. Applications to classification theory of Riemann surfaces are also given.

I would like to thank my advisor, Professor Hung-Hsi Wu, for his constant encouragement and many helpful suggestions.

1. Preliminaries. In this section we shall first fix notation, and give some definitions and formulas. All manifolds are assumed to be Riemannian. $\langle$,$\rangle denotes$ the Riemannian metric, and $|X|$ the length of the vector $X . D_{X} Y$ denotes the covariant derivative. The curvature tensor $R$ is defined by

$$
R(X, Y) Z=-D_{X} D_{Y} Z+D_{Y} D_{X} Z+D_{[X, Y]} Z
$$

thus $\langle R(X, Y) X, Y\rangle$ has the same sign as the sectional curvature of the plane spanned by $X$ and $Y$. The sign of the Laplacian is so chosen that in terms of local coordinates

$$
\Delta u=\sum_{i, j} \frac{1}{\sqrt{g}} \frac{\partial}{\partial x^{i}}\left(\sqrt{g} g^{i j} \frac{\partial u}{\partial x^{i}}\right),
$$

where $\left(g_{i j}\right)$ is the metric tensor, $g=\operatorname{det}\left(g_{i j}\right)$ and $\left(g^{i j}\right)=\left(g_{i j}\right)^{-1}$. Let $p$ be a point of $M$. We denote by $M_{p}$ the tangent space at $p$. $S_{p}$ denotes the set of unit tangent vectors at $p$. For a function $u$ defined near $p$, define the Hessian tensor $D^{2} u$ of $u$ at $p$ by

$$
\left(D^{2} u\right)(X, Y)=X(Y u)-\left(D_{X} Y\right) u,
$$

for $X, Y \in M_{p}$. In the above formula $X$ and $Y$ are arbitrarily extended near $p$, but it is easy to check $D^{2} u$ is a tensor, so it is independent of the extensions of $X$ and $Y$. Let $X_{1}, \ldots, X_{n}$ be an O.N. basis of $M_{p}$, then it is easy to prove using normal coordinates that

$$
\left.\Delta u\right|_{p}=\sum_{i=1}^{n}\left(D^{2} u\right)\left(X_{i}, X_{i}\right) .
$$

The above formula can be rephrased as follows: Take unit speed geodesics $\gamma_{i}$ such that $\gamma_{i}(0)=p$ and $\gamma_{i}^{\prime}(0)=X_{i}$ for $i=1,2, \ldots, n$. Then

$$
\left.\Delta u\right|_{p}=\left.\sum_{i=1}^{n} \frac{d^{2}}{d t^{2}} u\left(\gamma_{i}(t)\right)\right|_{t=0} .
$$

It is also well known that $\Delta u=\operatorname{div} \operatorname{grad} u$. Suppose $f: \mathbf{R} \rightarrow \mathbf{R}$ is a $C^{2}$ function. Then the following formula will be frequently used:

$$
\Delta f(u)=f^{\prime}(u) \Delta u+f^{\prime \prime}(u)|\operatorname{grad} u|^{2} .
$$

The distance between two points $p$ and $q$ of $M$ is denoted by $d(p, q)$. The following definition of convexity is very important. 
Definition 1.1. A continuous function $u: M \rightarrow \mathbf{R}$ is called convex if the restriction of $u$ to any geodesic is a convex function of the parameter $t$ of the geodesic. A set $S \subset M$ is called convex if any minimizing geodesic segment joining two points of $S$ lies entirely in $S$.

It is easy to see that in the case when $u$ is $C^{2}, u$ is convex if and only if $D^{2} u$ is positive semidefinite at every point.

The well-known Cartan-Hadamard theorem says that if $M$ is a simply connected, complete Riemannian manifold of nonpositive curvature (in this case $M$ is called a Cartan-Hadamard manifold), then the exponential map $\exp _{p}: M_{p} \rightarrow M$ is a diffeomorphism at any point $p \in M$. This motivates the following definition.

Definition 1.2. A point $p$ of a Riemannian manifold $M$ is called a pole, if the exponential map $\exp _{p}: M_{p} \rightarrow M$ is a diffeomorphism.

In order to define a Dirichlet problem, one needs a boundary. Even though the Cartan-Hadamard manifold has no boundary, we can define the boundary at infinity following Eberlein and O'Neill [EO]. For the rest of this section $M$ is assumed to be a Cartan-Hadamard manifold of dimension $n$.

Definition 1.3. Two unit speed geodesics $\alpha, \beta:(-\infty, \infty) \rightarrow M$ are called asymptotic if there exists a constant $c>0$ such that $d(\alpha(t), \beta(t)) \leqslant c$ for all $t \geqslant 0$.

The following facts are easy to check.

(1) If two unit speed asymptotic geodesics have a point in common, then they are the same.

(2) Given a geodesic $\alpha$ and a point $p \in M$, there exists a unique geodesic $\beta$ such that $\beta(0)=p$ and $\beta$ is asymptotic to $\alpha$.

(3) The asymptotic relation is an equivalence relation on the set of all geodesics in $M$; the equivalence classes are called the asymptotic classes. The asymptotic class of $\alpha$ is denoted by $\alpha(\infty)$, and $\alpha(-\infty)$ stands for the asymptotic class of the reverse curve of $\alpha$.

Definition 1.4. The boundary at infinity, or the Eberlein-O'Neill boundary of $M$ is the set $M(\infty)$ of all asymptotic classes of geodesics in $M$. Define $\bar{M}=M \cup M(\infty)$.

For any two points $p$ and $q$ of $M$, let $\gamma_{p q}$ denote the unique geodesic joining $p$ and $q$. Let $x \in M(\infty)$. Suppose there exists the unique geodesic $\gamma$ passing through $p$ and $\gamma(\infty)=x$, then we denote $\gamma$ by $\gamma_{p x}$. Let $x, y \in M(\infty), y \neq x$. We denote by $\gamma_{x y}$ the geodesic $\gamma$ such that $\gamma(-\infty)=x$ and $\gamma(\infty)=y$, if it exists and is unique. The existence and uniqueness of such geodesics are proved in [EO] under much weaker curvature assumptions than below.

THEOREM 1.5. If the Cartan-Hadamard manifold $M$ has the sectional curvature $\leqslant-c<0$, then for any $x, y \in \bar{M}$, there exists a unique geodesic $\gamma_{x y}$.

The following easy proposition indicates that the Eberlein-O'Neill boundary represents, in a sense, the set of all directions from a point.

Proposition 1.6. Let $p$ be any point of $M$. Then $M(\infty)$ and $S_{p}$ are in one-to-one correspondence, where $S_{p}$ is the set of unit tangent vectors at $p$.

Proof. For any $v \in S_{p}$, let $\gamma_{v}$ be the geodesic such that $\gamma_{v}(0)=p$ and $\gamma_{v}^{\prime}(0)=v$. It is easy to see that if $v \neq w$, then $\gamma_{v}$ and $\gamma_{w}$ are not asymptotic by the law of cosines. 
Conversely, let $\alpha$ be a geodesic in $M$. Let $v_{n} \in S_{p}$ be the tangent vector of the geodesic joining $p$ and $\alpha(n), n$ an integer. Then by Proposition 1.2 of [EO], $v_{n}$ converges to some $v$, and $\gamma_{v}(\infty)=\alpha(\infty)$.

Let $p$ be a point of $M$. For $v, w \in M_{p}$, define $\Varangle_{p}(v, w)$ to be the angle between $v$ and $w$ in the vector space $M_{p}$. If $x$ and $y \in \bar{M}$, then we define the angle $\Varangle_{p}(x, y)$ $=\Varangle_{p}\left(\gamma_{p x}^{\prime}(0), \gamma_{p y}^{\prime}(0)\right)$.

Definition 1.7. Let $p \in M, v \in S_{p}$ and $\delta>0$. Define the cone $\tilde{C}(v, \delta)$ in $M_{p}$ of opening angle $\delta$ and axis $v$ by

$$
\tilde{C}(v, \delta)=\left\{w \in M_{p} \Varangle_{p}(v, w)<\delta\right\} .
$$

The cone $C(v, \delta)$ in $\bar{M}$ is defined to be

$$
C(v, \delta)=\left\{x \in \bar{M} \mid \Varangle_{p}\left(v, \gamma_{p x}^{\prime}(0)\right)<\delta\right\} .
$$

Let $r>0$. We call $T(v, \delta, r)=C(v, \delta)-\{q \in M \mid d(p, q) \leqslant r\}$ the truncated cone of radius $r$ in $\bar{M}$.

Eberlein-O'Neill proved the following theorem.

THEOREM 1.8. Let $p \in M$. The set of all $T(v, \delta, r)$, for all $v \in S_{p}, \delta$ and $r>0$, and $B_{r}(q)=\{x \in M \mid d(q, x)<r\}$, for all $q \in M$, and $r>0$, defines a local basis of topology on $\bar{M}$, which is called the cone topology. The cone topology does not depend on the choice of $p$. Under this topology $\bar{M}$ is homeomorphic to the closed ball $\bar{B} \subset \mathbf{R}^{n}, M$ to the open ball $B$, and $M(\infty)$ to the boundary sphere $S^{n-1}=\partial \bar{B}$.

2. Asymptotic Dirichlet problem. Given a Cartan-Hadamard manifold we can pose the Dirichlet problem for harmonic functions in the following way.

Definition 2.1 (Asymptotic Dirichlet PROBlem). Let $M$ be a Cartan-Hadamard manifold. Given a continuous function $\phi: M(\infty) \rightarrow \mathbf{R}$, find a continuous function $u: \bar{M} \rightarrow \mathbf{R}$ such that $u$ is harmonic in $M$ and $\left.u\right|_{M(\infty)}=\phi$. Continuity is in terms of the cone topology.

For technical reasons which become clear in $\$ \S 3$ and 5, we shall rephrase the Dirichlet problem on a manifold with a pole. Throughout this section $M$ is merely assumed to be a manifold with a fixed pole $p$ with $\operatorname{dim} M=n$.

Definition 2.2. Given $v \in S_{p}$, the truncated cone in $M, K(v, \delta, r)$, is defined to be $T(v, \delta, r) \cap M=\left\{q \in M \mid d(p, q)>r\right.$ and $\left.\Varangle_{p}\left(v, \gamma_{p q}^{\prime}(0)\right)<\delta\right\}$. Suppose $u$ is a function defined only on $M$. We say $u$ converges to a number $A$ as $q \rightarrow \gamma_{v}(\infty)$ if given $\varepsilon>0$, there exist some $\delta>0$ and $r>0$ such that $|u(q)-A| \leqslant \varepsilon$ for all $q \in$ $K(v, \delta, r)$.

Definition 2.3 (Asymptotic Dirichlet PRoblem W.R.T. A POle $p$ ). Let $\phi: S_{p} \rightarrow \mathbf{R}$ be a continuous function, where $S_{p}$ is the set of unit tangent vectors at $p$. Find a harmonic function $u: M \rightarrow \mathbf{R}$ such that for every $v \in S_{p}, u(q)$ converges to $\phi(v)$ as $q \rightarrow \gamma_{v}(\infty)$ in the sense of Definition 2.2.

Even though the Eberlein-O'Neill boundary furnishes us with something tangible, it is rather cumbersome to work with in many concrete situations. Our Definitions 2.2 and 2.3 have the merit of avoiding $M(\infty)$ altogether by enabling us to deal only with $M$, because all we need is the concept of convergence in terms of truncated cones in $M$. This also saves us the trouble of redefining $M(\infty)$ in the presence of 
positive curvature, even if it could be done easily. In what follows, the convergence to the boundary values and the asymptotic Dirichlet problem itself should be understood in the sense of Definitions 2.2 an 2.3. In case $M$ is a Cartan-Hadamard manifold, if the Dirichlet problem is solvable in the sense of Definition 2.3, then we can extend $u$ to $\bar{M}$ as a continuous function. It is trivial to see that the two definitions are equivalent.

Our approach to this problem employs the classical Perron method of taking the upper envelope of the family of subharmonic functions with boundary values less than the given one.

Definition 2.4. A $C^{2}$ function $u: M \rightarrow \mathbf{R}$ is called harmonic if $\Delta u=0$. A continuous function $u: M \rightarrow \mathbf{R}$ is called subharmonic if for any ball $B \subset M$ and a harmonic function $h,\left.u\right|_{\partial B} \leqslant\left. h\right|_{\partial B}$ implies $u \leqslant h$ in $B$. We can similarly define superharmonic functions by reversing the above inequalities. It is well known that a $C^{2}$ function $u$ is subharmonic (resp. superharmonic) if and only if $\Delta u \geqslant 0$ (resp. $\Delta u \leqslant 0)$.

From the well-known maximum principle of E. Hopf, a subharmonic function cannot attain an interior maximum unless it is a constant function, i.e., the maximum of a subharmonic function occurs at the boundary. We have a similar result for the asymptotic case. In what follows, lim sup and lim inf are defined in terms of truncated cones as in Definition 2.2. Notation from Definitions 2.2 and 2.3 is used throughout this section.

Proposition 2.5 (Asymptotic MAXIMUM PRINCIPLE). (1) Suppose $f: M \rightarrow \mathbf{R}$ is $a$ subharmonic function such that $\lim \sup _{q \rightarrow \gamma_{v}(\infty)} f(q) \leqslant 0$ for all $v \in S_{p}$. Then $f \leqslant 0$ on M.

(2) If $f$ is a subharmonic function in $M$, and $g$ is a superharmonic function on $M$ such that $\lim \sup _{q \rightarrow \gamma_{v}(\infty)} f(q) \leqslant \lim \sup _{q \rightarrow \gamma_{v}(\infty)} g(q)$ for all $v \in S_{p}$. Then $f \leqslant g$ on $M$.

Proof of (1). Suppose there is a point $x \in M$ such that $f(x)=2 \varepsilon>0$. Let $l=d(p, x)$. By assumption, for any $v \in S_{p}$, there exist $\delta(v)>0$ and $r(v) \geqslant 2 l$ such that $f(q) \leqslant \varepsilon$ for all $q \in K(v, \delta, r)$. Let $U_{v}=\left\{w \in S_{p} \mid \Varangle_{p}(w, v)<\delta(v)\right\}$, then $\left\{U_{v}\right\}_{v \in S_{p}}$ is an open covering of $S_{p}$. By compactness we can choose finitely many $U_{v_{1}}, \ldots, U_{v_{k}}$ which cover $S_{p}$. Let $R=\max \left\{r\left(v_{i}\right): i=1, \ldots, k\right\}$. Then it is clear that $f(q) \leqslant \varepsilon$ for $q \in M$ such that $d(p, q) \geqslant R$. Now $x \in B_{R}(p)$ and $\left.f\right|_{\partial B_{R}} \leqslant \varepsilon$ and $f(q)=2 \varepsilon$. This contradicts the classical maximum principle. Applying (1) to the subharmonic function $f-g$, we get (2).

Define $F=\left\{f: M \rightarrow \mathbf{R} \mid f\right.$ is subharmonic and $\lim \sup f(q) \leqslant \phi(v)$ as $q \rightarrow \gamma_{v}(\infty)$ for all $v \in S_{p}$. Notice $F \neq \varnothing$. Define $u$ to be the upper envelope of this family $F$, i.e., $u(q)=\sup \{f(q) \mid f \in F\}$. It is well known that $u$ is a harmonic function (see Theorems 2.12 and 6.11 of [GT]). The main problem is how to prove that $u$ takes the asymptotically defined boundary values. The classical tool is the concept of barrier. Since this is the most important ingredient of our method, we would like to discuss the subtlety concerning our definition of barrier. Classically the Dirichlet problem is posed on the bounded domain $\Omega \subset \mathbf{R}^{n}$, and the barrier at a boundary point $x \in \partial \Omega$ is defined to be a subharmonic function $B$ on $\Omega$ such that $\lim _{q \rightarrow x} B(q)=0$ and $\lim \sup _{q \rightarrow y} B(q)<0$ for $y \neq x, y \in \partial \Omega$ (see [AS, p. 139]). This is a very general 
definition that does not require the continuity on the boundary. While this definition is good enough for the solution of the Dirichlet problem in the rotationally symmetric case, it is technically very difficult to construct a single function that can serve as a barrier (see the discussion at the beginning of §4). In order to overcome this difficulty we modify the definition of barrier slightly so that it may also depend on a small neighborhood of $x \in \partial \Omega$. This slight change turns out to play a crucial role in our case. Appropriate for our asymptotic Dirichlet problem is the following definition.

Definition 2.6. Given $v \in S_{p}, \delta>0, B: M \rightarrow \mathbf{R}$ is called a barrier at $v$ with angle $\delta$, if $B$ satisfies the following three conditions:

(1) $B$ is subharmonic on $M$,

(2) $B \leqslant 0$, and $\lim B(q)=0$ as $q \rightarrow \gamma_{v}(\infty)$,

(3) $\exists \eta>0$ such that $\lim \sup B(q) \leqslant-\eta$ as $q \rightarrow \gamma_{w}(\infty)$ for any $w \in S_{p}$ with $\Varangle_{p}(v, w)>\delta$.

THEOREM 2.7. Suppose, for any $v \in S_{p}$ and any sufficiently small $\delta>0$, there is a barrier $B$ at $v$ with angle $\delta$. Then $\lim u(q)=\phi(v)$ as $q \rightarrow \gamma_{v}(\infty)$ for any $v \in S_{p}$.

Proof. Fix any $v \in S_{p}$. Given $\varepsilon>0$, there exists a sufficiently small $\delta>0$ such that $|\phi(w)-\phi(v)| \leqslant \varepsilon$ for $\Varangle_{p}(w, v) \leqslant \delta$, since $\phi$ is continuous on $S_{p}$. Let $B$ be the barrier at $v$ with angle $\delta$, and let $\eta$ be the constant in Definition 2.6. Let $A$ be the supremum of $|\phi|$ on $S_{p}$. Choose $k>0$ such that $k \eta>2 A . \phi(v)+\varepsilon-k B$ is superharmonic, and we will prove that

$$
\liminf _{q \rightarrow \gamma_{w}(\infty)}\{\phi(v)+\varepsilon-k B(q)\} \geqslant \phi(w), \text { for all } w \in S_{p} .
$$

For $w \in S_{p}$ such that $\Varangle_{p}(w, v) \leqslant \delta,|\phi(w)-\phi(v)| \leqslant \varepsilon$ and $k B \leqslant 0$; thus $\phi(v)+\varepsilon$ $-k B \geqslant \phi(w)$. For $w \in S_{p}$ such that $\Varangle_{p}(w, v)>\delta$,

$$
\begin{aligned}
\liminf _{q \rightarrow \gamma_{w}(\infty)}\{\phi(v)+\varepsilon-k B(q)\} & =\phi(v)+\varepsilon-k \limsup _{q \rightarrow \gamma_{w}(\infty)} B(q) \\
& \geqslant \phi(v)+\varepsilon+k \eta \geqslant \phi(v)+\varepsilon+2 A \\
& \geqslant \phi(v)+\varepsilon+\phi(w)-\phi(v) \geqslant \phi(w) .
\end{aligned}
$$

We now claim that

$$
\limsup _{q \rightarrow \gamma_{w}(\infty)}\{\phi(v)-\varepsilon+k B(q)\} \leqslant \phi(w), \quad \text { for all } w \in S_{p} .
$$

For $w \in S_{p}$ such that $\Varangle_{p}(v, w) \leqslant \delta, \phi(v)-\varepsilon \leqslant \phi(w)$ and $k B \leqslant 0$, so $\phi(v)-\varepsilon+$ $k B \leqslant \phi(w)-\varepsilon \leqslant \phi(w)$. For $w \in S_{p}$ such that $\Varangle_{p}(u, w)>\delta$,

$$
\begin{aligned}
\limsup _{q \rightarrow \gamma_{w}(\infty)}\{\phi(v)-\varepsilon+k B(q)\} & \leqslant \phi(v)-\varepsilon-k \eta \leqslant \phi(v)-2 A \\
& \leqslant \phi(v)+\phi(w)-\phi(v)=\phi(w) .
\end{aligned}
$$

From (*) and Proposition 2.5, we get $\phi(v)+\varepsilon-k B \geqslant f$, for any $f \in F$. Thus we have $\phi(v)+\varepsilon-k B \geqslant u$. From $(* *)$, we have $\phi(v)-\varepsilon+k B \in F$, so by the definition of $u$, we get $u \geqslant \phi(v)-\varepsilon+k B$. Combining these two inequalities, we get $|u-\phi(v)| \leqslant \varepsilon-k B$. 
Letting $q \rightarrow \gamma_{v}(\infty)$, we get

$$
\limsup _{q \rightarrow \gamma_{v}(\infty)}|u(q)-\phi(v)| \leqslant \varepsilon-k \lim _{q \rightarrow \gamma_{v}(\infty)} B(q)=\varepsilon
$$

in the sense of Definition 2.2. Since $\varepsilon$ is arbitrary, we get $\lim _{q \rightarrow \gamma_{t}(\infty)} u(q)=\phi(v)$.

COROLlARY 2.8. If the assumption of Theorem 2.7 is satisfied, then the asymptotic Dirichlet problem is solvable, hence $M$ possesses nonconstant bounded harmonic functions by Proposition 2.5.

EXAMPLE 2.9. At this point one might wonder why we insist on the convergence to the boundary values in terms of truncated cones. Actually one may define the convergence in terms of rays, i.e., the radial limit. But then this violates the asymptotic maximum principle as the following example shows: Let $M$ be the usual upper half-plane of $\mathbf{C}$ equipped with the Poincare metric. Fix a point $p=(0,1)$. Geodesic rays from $p$ are circles through $p$ meeting with the $x$ axis perpendicularly or the vertical line through $(0,1)$. Let $u(x, y)=e^{x} \sin y$, then the lim sup of the radial limit through each ray is at most 1 , but $u$ can be as big as we wish. Or let $v(x, y)=x y$, then the radial limits are all zero but $v>0$ in $M$. Clearly $u$ and $v$ are harmonic in $M$. The last example $v(x, y)=x y$ is due to T. Wolff. Fatou's theorem says that every bounded harmonic function on the open disk has a radial limit in almost every direction, but it may not have boundary values in the usual sense. This also shows that the radial limits do not reflect the overall behavior very well.

3. Rotationally symmetric manifolds. Liouville's theorem for harmonic functions in $\mathbf{R}^{n}$ tells us that the asymptotic Dirichlet problem is not solvable for $\mathbf{R}^{n}$. Furthermore from the recent result of Greene and Wu [GW2], if the curvature stays "close" to zero, then the asymptotic Dirichlet problem cannot be solved, since $M$ is again isometric to $\mathbf{R}^{n}$ when $n \geqslant 3$. On the other hand Theorem 0.1 suggests that the asymptotic Dirichlet problem may be solvable under the curvature decay condition of Theorem 0.1(A). The purpose of this section is to show that this is the case under the additional assumption of rotational symmetry. One should note the sharpness of the curvature conditions distinguishing the two cases of Theorem 0.1.

Definition 3.1. Let $M$ be a manifold with a pole $p . M$ is called rotationally symmetric at $p$, if every linear isometry $\phi: M \rightarrow M$ is realized as the differential of an isometry $\Phi: M \rightarrow M$, i.e., $\Phi(p)=p$ and $\Phi^{*}(p)=\phi$. In other words the isotropy subgroup at $p$ of the isometry group of $M$ is $O(n)$. Greene and Wu [GW1, p. 24] call this manifold a model.

Throughout this section $M$ is assumed to be a Riemannian manifold with a fixed pole $p$, rotationally symmetric at $p$, with $\operatorname{dim} M=n$.

Definition 3.2. Let $q$ be a point in $M, p \neq q$. The two-dimensional subspace $\pi$ of $M_{q}$ is called a radial plane, if $\pi$ is tangent to the geodesic $\gamma_{p q}$ joining $p$ and $q$. The radial curvature is the restriction of the sectional curvature to radial planes.

Since $M$ is rotationally symmetric at $p$, it is easy to see that the Riemannian metric $d s^{2}$ on $M-\{0\}$ can be written as $d s^{2}=d r^{2}+f(r)^{2} d \Theta^{2}$ in terms of geodesic polar coordinates, where $r$ is the distance function from $p$, and $\Theta$ and $d \Theta^{2}$ are the local 
coordinates and the metric of the standard sphere $S^{n-1}$. Throughout this section $r$ always denotes the geodesic distance function from $p$.

In view of $\$ 2$ all we have to do to solve the Dirichlet problem is to construct a barrier at fixed $v \in S_{p}$ at any angle. From now on $v \in S_{p}$ is held fixed. Define the angle function $\theta$ w.r.t. $\gamma_{v}$ to be

$$
\theta(q)=\Varangle_{p}\left(\gamma_{p q}^{\prime}(0), \gamma_{v}^{\prime}(0)\right)
$$

for all $q \in M$. The value of $\theta$ is taken to be $0 \leqslant \theta \leqslant \pi$. Notice that $\pi$ is $C^{\infty}$ on $M-\left\{q \mid q=\gamma_{v}(t),-\infty<t<\infty\right\}$. Furthermore, $\cos \theta$ is $C^{\infty}$ on $M-\{p\}$, since in terms of normal coordinates $x^{1}, \ldots, x^{n}$ at $p$,

$$
\cos \theta=\frac{x^{1}}{\sqrt{\left(x^{1}\right)^{2}+\cdots+\left(x^{n}\right)^{2}}}
$$

where the positive $x^{1}$ coordinate axis is the geodesic ray $\gamma_{v}(t)$. Let $\theta_{2}, \ldots, \theta_{n}$ be the spherical angles on $S^{n-1}$ such that $\theta_{2}=\theta$. Then the polar coordinates and the normal coordinates are related by the equations

$$
\begin{aligned}
x^{1} & =r \cos \theta_{2}, \\
x^{2} & =r \sin \theta_{2} \cos \theta_{3}, \\
\vdots & \\
x^{n-1} & =r \sin \theta_{2} \sin \theta_{3} \cdots \cos \theta_{n}, \\
x^{n} & =r \sin \theta_{2} \sin \theta_{3} \cdots \sin \theta_{n} .
\end{aligned}
$$

By computing the metric $d \Theta^{2}$ on the standard sphere in terms of spherical angles, we get the Riemannian metric on $M$

$$
d s^{2}=d r^{2}+f(r)^{2}\left\{d \theta_{2}^{2}+\left(\sin \theta_{2} d \theta_{3}\right)^{2}+\cdots+\left(\sin \theta_{2} \cdots \sin \theta_{n-1} d \theta_{n}\right)^{2}\right\} .
$$

Proposition 3.3. Let $M$ be a Riemannian manifold with a pole $p$. Suppose the Riemannian metric in terms of geodesic polar coordinates at $p$ is of the form

$$
d s^{2}=d r^{2}+f\left(r, \theta_{2}, \ldots, \theta_{n}\right)^{2}\left\{d \theta_{2}^{2}+\left(\sin \theta_{2} d \theta_{3}\right)^{2}+\cdots+\left(\sin \theta_{2} \cdots \sin \theta_{n-1} d \theta_{n}\right)^{2}\right\} .
$$

Then

$$
\Delta \theta=(n-3) \frac{1}{f^{3}} \frac{\partial f}{\partial \theta}+(n-2) \frac{1}{f^{2}} \frac{\cos \theta}{\sin \theta} \quad \text { where } \theta \neq 0, \pi
$$

and

$$
\Delta \cos \theta=-(n-3) \sin \theta \frac{1}{f^{3}} \frac{\partial f}{\partial \theta}-(n-1) \frac{1}{f^{2}} \cos \theta \text { on } M-\{p\} .
$$

In particular if $M$ is rotationally symmetric at $p$, then $\Delta \theta=(n-2) \cos \theta / f^{2} \sin \theta$, where $\theta \neq 0, \pi$ and $\Delta \cos \theta=-(n-1) \cos \theta / f^{2}$ on $M-\{p\}$.

(Note that we defined $\theta_{2}=\theta$.)

Proof. Note that $\theta$ is not differentiable where $\theta=0$ or $\pi$. But since $\cos \theta$ is $C^{\infty}$ on $M-\{p\}, \Delta \cos \theta$ computed where $\theta \neq 0, \pi$ can be extended to $M-\{p\}$. We 
will use the formula $\Delta u=\operatorname{div} \operatorname{grad} u$. Pick the orthonormal frame $X_{1}, \ldots, X_{n}$, such that

$$
X_{1}=\frac{\partial}{\partial r}, X_{2}=\frac{1}{f} \frac{\partial}{\partial \theta_{2}}, \ldots, X_{n}=\frac{1}{f \sin \theta_{2} \cdots \sin \theta_{n-1}} \frac{\partial}{\partial \theta_{n}} .
$$

Now $\operatorname{grad} \theta=\operatorname{grad} \theta_{2}=\left(1 / f^{2}\right) \partial / \partial \theta_{2}$. Then

$$
\begin{aligned}
& \Delta \theta=\operatorname{div} \operatorname{grad} \theta=\sum_{i=1}^{n}\left\langle D_{X_{i}} \frac{1}{f^{2}} \frac{\partial}{\partial \theta_{2}}, X_{i}\right\rangle, \\
& i=1 ;\left\langle D_{X_{1}} \frac{1}{f^{2}} \frac{\partial}{\partial \theta_{2}}, X_{1}\right\rangle=X_{1}\left\langle\frac{1}{f^{2}} \frac{\partial}{\partial \theta_{2}}, X_{1}\right\rangle-\left\langle\frac{1}{f^{2}} \frac{\partial}{\partial \theta_{2}}, D_{X_{1}} X_{1}\right\rangle=0 \text {, } \\
& i=2 ; \quad\left\langle D_{X_{2}} \frac{1}{f^{2}} \frac{\partial}{\partial \theta_{2}}, X_{2}\right\rangle=\left\langle D_{(1 / f) \partial / \partial \theta_{2}} \frac{1}{f^{2}} \frac{\partial}{\partial \theta_{2}}, \frac{1}{f} \frac{\partial}{\partial \theta_{2}}\right\rangle \\
& =\frac{1}{f^{2}}\left\langle D_{\partial / \partial \theta_{2}} \frac{1}{f^{2}} \frac{\partial}{\partial \theta_{2}}, \frac{\partial}{\partial \theta_{2}}\right\rangle \\
& =\frac{1}{f^{2}} \frac{\partial}{\partial \theta_{2}}\left\langle\frac{1}{f^{2}} \frac{\partial}{\partial \theta_{2}}, \frac{\partial}{\partial \theta_{2}}\right\rangle-\frac{1}{f^{4}}\left\langle\frac{\partial}{\partial \theta_{2}}, D_{\partial / \partial \theta_{2}} \frac{\partial}{\partial \theta_{2}}\right\rangle \\
& =\frac{-1}{2 f^{4}} \frac{\partial}{\partial \theta_{2}}\left\langle\frac{\partial}{\partial \theta_{2}}, \frac{\partial}{\partial \theta_{2}}\right\rangle \text {, since }\left\langle\frac{1}{f^{2}} \frac{\partial}{\partial \theta_{2}}, \frac{\partial}{\partial \theta_{2}}\right\rangle \equiv 1 \\
& =\frac{-1}{2 f^{4}} \frac{\partial}{\partial \theta_{2}} f^{2}=-\frac{1}{f^{3}} \frac{\partial f}{\partial \theta_{2}}, \\
& i \geqslant 3 ; \quad\left\langle D_{X_{i}} \frac{1}{f^{2}} \frac{\partial}{\partial \theta_{2}}, X_{i}\right\rangle=\frac{1}{\left(f \sin \theta_{2} \cdots \sin \theta_{i-1}\right)^{2}}\left\langle D_{\partial / \partial \theta_{i}} \frac{1}{f^{2}} \frac{\partial}{\partial \theta_{2}}, \frac{\partial}{\partial \theta_{i}}\right\rangle \\
& =\frac{1}{\left(f \sin \theta_{2} \cdots \sin \theta_{i-1}\right)^{2}}\left[\frac{-2}{f^{3}} \frac{\partial f}{\partial \theta_{i}}\left\langle\frac{\partial}{\partial \theta_{2}}, \frac{\partial}{\partial \theta_{i}}\right\rangle+\frac{1}{f^{2}}\left\langle D_{\partial / \partial \theta_{i}} \frac{\partial}{\partial \theta_{2}}, \frac{\partial}{\partial \theta_{i}}\right\rangle\right] \\
& =\frac{1}{f^{4}\left(\sin \theta_{2} \cdots \sin \theta_{i-1}\right)^{2}}\left\langle D_{\partial / \partial \theta_{i}} \frac{\partial}{\partial \theta_{2}}, \frac{\partial}{\partial \theta_{i}}\right\rangle, \text { since }\left\langle\frac{\partial}{\partial \theta_{2}}, \frac{\partial}{\partial \theta_{i}}\right\rangle=0 \\
& =\frac{1}{f^{4}\left(\sin \theta_{2} \cdots \sin \theta_{i-1}\right)^{2}} \cdot \frac{1}{2} \frac{\partial}{\partial \theta_{2}}\left\langle\frac{\partial}{\partial \theta_{i}}, \frac{\partial}{\partial \theta_{i}}\right\rangle \\
& =\frac{1}{f^{4}\left(\sin \theta_{2} \cdots \sin \theta_{i-1}\right)^{2}} \cdot \frac{1}{2} \frac{\partial}{\partial \theta_{2}}\left(f \sin \theta_{2} \cdots \sin \theta_{i-1}\right)^{2} \\
& =\frac{1}{f^{3}} \frac{\partial f}{\partial \theta_{2}}+\frac{1}{f^{2}} \frac{\cos \theta_{2}}{\sin \theta_{2}} \text {. }
\end{aligned}
$$

Thus we get

$$
\Delta \theta=(n-3) \frac{1}{f^{3}} \frac{\partial f}{\partial \theta}+(n-2) \frac{1}{f^{2}} \frac{\cos \theta}{\sin \theta}
$$


Hence

$$
\begin{aligned}
\Delta \cos \theta & =-\sin \theta \Delta \theta-\cos \theta|\operatorname{grad} \theta|^{2} \\
& =-(n-3) \sin \theta \frac{1}{f^{3}} \frac{\partial f}{\partial \theta}-(n-1) \frac{1}{f^{2}} \cos \theta \quad \text { Q.E.D. }
\end{aligned}
$$

As mentioned earlier our job here is to construct a barrier at $v \in S_{p}$. The function $\cos \theta$ serves as a good candidate for the barrier, except that is is not subharmonic. To compensate for this lack of subharmonicity without hurting the asymptotic boundary behavior we can add a function $g(r)$ of $r$ which goes to zero as $r \rightarrow \infty$. To prove this it is necessary to estimate $\Delta r$ under the given curvature assumption. Fortunately we have a very simple expression of $\Delta r$ in terms of $f(r)$, namely $\Delta r=(n-1) f^{\prime}(r) / f(r)$ (cf. [GW1, p. 30]). Since $M$ is rotationally symmetric at $p$, any radial plane at any $q \in M$ can get mapped into any other radial plane by an isometry. Also given two points $q_{1}$ and $q_{2}$ with the same distance from $p$, we can find an isometry $\Phi$ such that $\Phi\left(q_{1}\right)=q_{2}$. Thus it is trivial that the radial curvature depends only on the distance $r$ from $p$, i.e., we have the radial curvature function $K(r) . K(r)$ and $f(r)$ are related by the equation $f^{\prime \prime}(r)+K(r) f(r)=0, f(0)=0$ and $f^{\prime}(0)=1$. (See [GW1, p. 30] for relevant discussions on this.)

PROPOSITION 3.4. Suppose $f:[a, \infty) \rightarrow \mathbf{R}$ is a positive strictly increasing function of $r$ satisfying the equation $f^{\prime \prime}(r)+K(r) f(r)=0$, where $K(r) \leqslant-A / r^{2} \log r$, for some $A>1$ on $[a, \infty)$. Then for any $B, 1<B<A$, there exists some $r_{0} \geqslant a$ such that for all $r \geqslant r_{0}$

$$
f(r) \geqslant r(\log r)^{B}, \quad \frac{f^{\prime}(r)}{f(r)} \geqslant \frac{1}{r}+\frac{B}{r \log r} .
$$

To prove this proposition, we need the following lemma. Part (1) is due to Milnor [Mi] and part (2) is the well-known Sturm comparison theorem.

LEMMA 3.5. Let $h$ and $k$ be positive strictly increasing functions on $[a, \infty)$.

(1) If $h(a)<k(a), h^{\prime}(a)<k^{\prime}(a)$ and $h^{\prime \prime} / h \leqslant k^{\prime \prime} / k$ on $[a, \infty)$, then $h<k$ on $[a, \infty)$.

(2) If $h^{\prime \prime} / h \leqslant k^{\prime \prime} / k$ and $h^{\prime}(a) / h(a) \leqslant k^{\prime}(a) / k(a)$, then $h^{\prime}(r) / h(r) \leqslant k^{\prime}(r) / k(r)$ for all $r \in[a, \infty)$.

Proof of Proposition 3.4. Our argument is based on that of Greene and $\mathrm{Wu}$ [GW 1, pp. 54-55]. Choose any $B_{1}$ such that $B<B_{1}<A$. Define $g_{1}(r)=C r(\log r)^{B_{1}}$, $C$ is a constant to be determined later. After easy computations, we get

$$
\frac{g_{1}^{\prime \prime}}{g_{1}}=\frac{B_{1}}{r^{2} \log r}\left[1+\frac{B_{1}-1}{\log r}\right] \text {. }
$$

Since $B_{1}<A$, there exists some $r_{1} \geqslant a$ such that for all $r \geqslant r_{1}$,

$$
\frac{g_{1}^{\prime \prime}}{g_{1}} \leqslant \frac{A}{r^{2} \log r} \leqslant-K(r)=\frac{f^{\prime \prime}}{f} .
$$


Choose $C$ small enough so that $g_{1}\left(r_{1}\right)<f\left(r_{1}\right)$ and $g_{1}^{\prime}\left(r_{1}\right)<f^{\prime}\left(r_{1}\right)$, then by (1), we have

$$
f(r) \geqslant g_{1}(r)=C r(\log r)^{B_{1}}
$$

for all $r \geqslant r_{1}$. Let us define $g(r)=r(\log r)^{B}$, then since $B<B_{1}$, it is obvious that for $r \geqslant r_{1}$

$$
\frac{g^{\prime \prime}}{g}=\frac{B}{r^{2} \log r}\left[1+\frac{B-1}{\log r}\right] \leqslant \frac{A}{r^{2} \log r} \leqslant \frac{f^{\prime \prime}}{f} .
$$

Suppose that for all $r \geqslant r_{1}$,

$$
\frac{f^{\prime}(r)}{f(r)}<\frac{1}{r}+\frac{B}{r \log r}=\frac{g^{\prime}(r)}{g(r)},
$$

then by integrating the above inequality from $r_{1}$ to $r$, then taking the exponential, we get, for some constant $C_{1}>0, f(r)<C_{1} r(\log r)^{B}$ for all $r \geqslant r_{1}$. But since $B<B_{1}$, this is a contradiction to the inequality $(*)$. Thus there must exist some $r_{2} \geqslant r_{1}$ such that

$$
\frac{f^{\prime}\left(r_{2}\right)}{f\left(r_{2}\right)} \geqslant \frac{1}{r_{2}}+\frac{B}{r_{2} \log r_{2}}=\frac{g^{\prime}\left(r_{2}\right)}{g\left(r_{2}\right)} .
$$

Applying (2) of Lemma 3.5, we can then conclude that

$$
\frac{f^{\prime}(r)}{f(r)} \geqslant \frac{1}{r}+\frac{B}{r \log r}
$$

for all $r \geqslant r_{2}$. Now since $B_{1}>B$, there exists some $r_{0} \geqslant r_{2}$ such that $\operatorname{Cr}(\log r)^{B_{1}} \geqslant$ $r(\log r)^{B}$.

We are now ready to prove the main theorem of this section.

THEOREM 3.6. Let $M$ be a rotationally symmetric manifold with a pole $p$ of dimension $n \geqslant 2$. Suppose the radial curvature $\leqslant-A / r^{2} \log r$, for some $A>1$, outside $a$ compact set. Then the asymptotic Dirichlet problem with respect to the pole $p$ as posed in Definition 2.3 is solvable, hence $M$ possesses nonconstant bounded harmonic functions.

Proof. All we have to to is to construct a barrier at $v \in S_{p}$, then since $v$ is arbitrary we have a barrier at every $w \in S_{p}$. Let us define $\beta_{1}=\cos \theta-(\log r)^{-\varepsilon}-1$, where $\varepsilon$ is a constant to be chosen later, which depends only on $A$. Now

$$
-\Delta(\log r)^{-\varepsilon}=\varepsilon(\log r)^{-\varepsilon-1} \frac{1}{r} \Delta r-\varepsilon(\varepsilon+1)(\log r)^{-\varepsilon-2} \frac{1}{r^{2}}-\varepsilon(\log r)^{-\varepsilon-1} \frac{1}{r^{2}} .
$$

By Proposition 2.20 of [GW1, p. 30], we have

$$
\Delta r=(n-1) f^{\prime}(r) / f(r) \geqslant f^{\prime}(r) / f(r) .
$$

Assume $B$ and $r_{0}$ are the same as in Proposition 3.4, then by combining the above two inequalities and Proposition 3.4, we have

$$
-\Delta(\log r)^{-\varepsilon} \geqslant \frac{\varepsilon(B-\varepsilon-1)}{r^{2}(\log r)^{2+\varepsilon}}
$$


for all $r \geqslant r_{0}$. On the other hand, by Propositions 3.3 and 3.4, we have

$$
\Delta \cos \theta=\frac{-(n-1) \cos \theta}{f^{2}} \geqslant \frac{-(n-1)}{r^{2}(\log r)^{2 B}}
$$

for all $r \geqslant r_{0}$. Choose $\varepsilon$ such that $0<\varepsilon<B-1$. Then it is easy to see that there exists some $R \geqslant r_{0}$ such that $\Delta \beta_{1} \geqslant 0$ at every point $q$ such that $d(p, q) \geqslant R$. Let $\Omega_{1}=\{q \in M \mid r(q)>2 R$ and $\theta(q)<\pi / 4\}$ and $\Omega_{2}=\{q \in M \mid r(q) \geqslant 3 R$ and $\theta(q)$ $\leqslant \pi / 6\}$. Let $a=\sup \left\{\beta_{1}(q) \mid q \in \Omega_{1}-\Omega_{2}\right\}$. It is easy to see that $a<0$. Let us now define $\beta$ in $\Omega_{1}$ such that $\beta(q)=\max \left\{a, \beta_{1}(q)\right\}$ for all $q \in \Omega_{1}$. Clearly $\beta \equiv a$ on the open set $\Omega_{1}-\Omega_{2}$, and $\beta$ is subharmonic on $\Omega_{1}$, since the maximum of two subharmonic functions is again subharmonic. Because $\beta \equiv a$ on $\Omega_{1}-\Omega_{2}, \beta$ extends to a constant function on $M-\Omega_{1}$. Obviously $\beta$ is again subharmonic on $M$. Hence $\beta$ is the desired barrier at $v$ with any angle $\delta<\pi / 6$.

We will give the following characterization of rotationally symmetric manifolds. One is referred to [Ci2] for a more general result that allows $M$ to be compact.

THEOREM 3.7. Let $M$ be a manifold with a fixed pole $p$ with $\operatorname{dim} M=n \geqslant 3$. Then the following are equivalent.

(1) $M$ is rotationally symmetric at $p$.

(2) The exponential image of any cone of opening angle $\leqslant \pi / 2$ in $M_{p}$ with any axis $v \in M_{p}$ is convex.

(3) The exponential image of any linear subspace of $M_{p}$ is totally geodesic.

(4) The radial curvature w.r.t. $p$ depends only on the distance $r$ from $p$.

(5) The Riemannian metric in terms of the geodesic polar coordinates is $d s^{2}=d r^{2}+$ $f(r)^{2} d \Theta^{2}$ for some function $f:[0, \infty) \rightarrow \mathbf{R}$, where $d \Theta^{2}$ is the metric of the standard sphere.

REMARK 3.8. The convexity condition in Theorem 3.7 cannot be improved to total convexity (recall that a set $S$ is totally convex if for any $q_{1}, q_{2} \in S$, any geodesic $\gamma$ joining $q_{1}$ and $q_{2}$ lies entirely in $S$ ) as the following examples shows: Take $S_{+}^{2}$, the upper hemisphere of the sphere, and $S^{1} \times[0, \infty)$, half of the infinite cylinder. Match their boundaries, and deform it so that they form a smooth manifold $M$ which is rotationally symmetric at the north pole of $S_{+}^{2}$. Take a maximal geodesic $\gamma$ through the north pole, then $\gamma$ divides $M$ into two components, $U_{1}$ and $U_{2}$. We may assume $U_{1} \cap$ cylinder $=\left\{\left(e^{i \theta}, r\right) \mid 0<\theta<\pi, r>0\right\}$ and $U_{2} \cap$ cylinder $=\left\{\left(e^{i \theta}, r\right) \mid\right.$ $\pi<2 \pi, r>0\}$, where $e^{i \theta} \in S^{1}$. Let $q_{1}$ and $q_{2}$ be points in $U_{1} \cap$ cylinder such that $q_{1}=\left(e^{i \pi / 3}, 1\right)$ and $q_{2}=\left(e^{i 2 \pi / 3}, 1\right)$. Then $\gamma:[\pi / 3,2 \pi / 3] \rightarrow M$ such that $\gamma(\theta)=$ $\left(e^{i \theta}, 1\right)$ is a minimizing geodesic in $U_{1}$. But $\gamma_{1}:[2 \pi / 3,7 \pi / 3] \rightarrow M$ such that $\gamma_{1}(\theta)=$ $\left(e^{i \theta}, 1\right)$ is another geodesic joining $q_{1}$ and $q_{2}$, but $\gamma_{1}$ meets with $U_{2}$.

\section{Appendix to §3.}

Proposition 3.9. Let $M$ be a manifold with a fixed pole p. Assume that the Riemannian metric restricted to every geodesic sphere is conformal to that of the standard sphere, namely, $d s^{2}$ is of the form

$d s^{2}=d r^{2}+f\left(r, \theta_{2}, \ldots, \theta_{n}\right)^{2}\left\{d \theta_{2}^{2}+\left(\sin \theta_{2} d \theta_{3}\right)^{2}+\cdots+\left(\sin \theta_{2} \cdots \sin \theta_{n-1} d \theta_{n}\right)^{2}\right\}$. 
Assume also that the radial curvature outside some compact set $\leqslant-A / r^{2} \log r$, for some $A>1$. If $\operatorname{dim} M=3$, or $\left|\partial f / \partial \theta_{2}\right| \leqslant C r \log r$, for some $C>0$, then the asymptotic Dirichlet problem w.r.t. $p$ is solvable. We denote $\theta_{2}=\theta$.

Proof. The proof is almost the same as that of Theorem 3.6. First we can certainly estimate $\Delta r$ using the Laplacian comparison theorem [GW1, p. 26] by comparing with the rotationally symmetric manifold. Now $\partial / \partial \theta_{2}$ is a Jacobi field along any geodesic ray from $p$ such that $\left|\partial / \partial \theta_{2}\right|=f$. Then using Proposition 3.4 it is not hard to conclude that for any $B, 1<B<A$, there exists some $r_{0}>0$ such that for all $r \geqslant r_{0}, f \geqslant r(\log r)^{B}$ and

$$
\Delta r \geqslant(n-1)\left\{\frac{1}{r}+\frac{B}{r \log r}\right\} \geqslant \frac{1}{r}+\frac{B}{r \log r} .
$$

If $n=3$, then $\Delta \cos \theta=-2 \cos \theta / f^{2} \geqslant-2 / r^{2}(\log r)^{2 B}$. If $\left|\partial f / \partial \theta_{2}\right| \leqslant C r \log r$, then there exists some $C_{1}$ such that

$$
\Delta \cos \theta \geqslant-C_{1}\left\{\frac{1}{r^{2}(\log r)^{3 B-1}}+\frac{1}{r^{2}(\log r)^{2 B}}\right\} .
$$

In either case the rest of the proof is verbatim the same as in Theorem 3.6.

4. Convex conic neighborhood condition. In this section we present a new approach to the construction of barrier which is conceptually very different from that we constructed in $\S 3$. In $\S 3$ the key point is that $\Delta \cos \theta$ is very well behaved under the assumption of rotational symmetry. But once the rotational symmetry assumption is dropped, the behavior of $\Delta \cos \theta$ cannot be controlled by bounding the sectional curvature. Here we will give a simple illustration. Let $M$ be a 2-dimensional Cartan-Hadamard manifold, then in terms of polar coordinates the Riemannian metric $d s^{2}=d r^{2}+f(r, \theta)^{2} d \theta^{2}$. The curvature $K=-\partial^{2} f / \partial r^{2} / f$, thus $K$ depends on the radial behavior of $f$. On the other hand $\operatorname{grad} \theta=\partial / f^{2} \partial \theta$, thus by simple computations similar to those given in the proof of Proposition 3.3 it is easy to check that $\Delta \theta=\operatorname{div} \operatorname{grad} \theta=-\left(1 / f^{3}\right) \partial f / \partial \theta$. Thus $\Delta \cos \theta=\sin \theta\left(1 / f^{3}\right) \partial f / \partial \theta-$ $\left(1 / f^{2}\right) \cos \theta$. Since the curvature depends only on the radial behavior of $f$, we can perturb $f$ in the angular direction to make $|\partial f / \partial \theta|$ arbitrarily large while the curvature of the perturbed metric remains as close as we wish to that of the original metric. Going back to the general case, if we take a $C^{2}$ function $\beta$ on $S_{p}$ and extend it to $M-\{p\}$ and try to check if $\beta$ is subharmonic we encounter a similar difficulty. The whole point is that angular behavior cannot be controlled by bounding the sectional curvature.

Our construction of the barrier is based on the idea of the barrier with angle which does not depend sharply on the angular behavior. We feel that one of our main contributions lies in reducing the question on the second derivatives of a function depending on angles to the convexity question. This kind of idea will be fully exploited in $\$ 5$. Let us first record two basic facts concerning convex sets, Theorem 1.1 and Theorem 4.2. Both results are more or less known to many people. It should be noted that $\mathrm{S}$. Alexander [Ax] proved Theorem 4.1. Theorems 4.1 and 4.2 
could be sharpened in terms of focal points and cut loci, but we will present them in a simpler form which we need.

THEOREM 4.1. Suppose $M$ be a Cartan-Hadamard manifold. Let $\Omega$ be a connected open set of $M$ with $C^{2}$ boundary $S=\partial \Omega$. Let $N$ be the unit outward normal vector field on $S$. Then $\bar{\Omega} \subset M$ is convex if and only if $\left\langle B_{X X}, N\right\rangle \leqslant 0$ for any vector $X$ tangent to $S$, where $B$ is the second fundamental form of $S$ given by $B_{X Y}=\left\langle D_{X} Y, N\right\rangle N$.

Proof. Suppose $\bar{\Omega}$ is convex. Let $p \in S$, and let $X \in M_{p}$ be a unit vector tangent to $S$. Choose normal coordinates $x^{1}, \ldots, x^{n}$ at $p$ such that $\partial / \partial x^{1}=N$ and $\partial / \partial x^{2}=X$ at $p$. Let $C(t)$ be the geodesic in $S$ in terms of the induced metric on $S$ such that $C(0)=p$ and $C^{\prime}(0)=X$. We claim $x^{1} \leqslant 0$ on $S$. Suppose there is a point $q \in S$ such that $x^{1}(q)>0$, then $\left\langle\gamma_{p q}^{\prime}(0), N\right\rangle>0$. Let $\alpha(w)$ be a geodesic in $M$ such that $\alpha(0)=p$ and $\alpha^{\prime}(0)=N$. Let $l=d(p, q)$. We can assume $\gamma_{p q}(t) \notin \bar{\Omega}$ for all $t$, $0<t<l$, since $\left\langle\gamma_{p q}^{\prime}(0), N\right\rangle>0$. Then for sufficiently small $w$, the geodesic ray emanating from $q$ to $\alpha(w)$ must meet with $S$ at some point $a$ beyond $\alpha(w)$. For otherwise by letting $w \rightarrow 0$ it is easy to see that $\gamma_{p q}$ is tangent to $S$ at $p$ which contradicts the fact $\left\langle\gamma_{p q}^{\prime}(0), N\right\rangle>0$. Then since $a$ and $q$ both lie in the convex set $\bar{\Omega}$, $\alpha(w) \in \bar{\Omega}$ which is absurd. Thus $x^{1} \leqslant 0$ on $S$. Therefore we have $x^{1}(C(t)) \leqslant 0$ and $x^{1}(C(0))=0$ which imply that $\left.\left(d^{2} / d t^{2}\right) x^{1}(C(t))\right|_{t=0} \leqslant 0$. So the Hessian $D^{2} x^{1}(X, X)=\left(d^{2} / d t^{2}\right) x^{1}(C(t))-\left\langle D_{X} X, \operatorname{grad} x^{1}\right\rangle \leqslant-\left\langle B_{X X}, N\right\rangle$ at $p$. On the other hand, let $\beta(t)$ be the geodesic in $M$ such that $\beta(0)=p$ and $\beta^{\prime}(0)=X$, then since all the Christoffel symbols vanish at $q, D^{2} x^{1}(X, X)=\partial^{2} x^{1} /\left(\partial x^{2}\right)^{2}=0$ at $q$. Thus we proved $\left\langle B_{X X}, N\right\rangle \leqslant 0$. Conversely assume $\left\langle B_{X X}, N\right\rangle \leqslant 0$, then R. Bishop [B] proved that $\bar{\Omega}$ is locally convex, i.e., for any point $q \in \bar{\Omega}$, there exists a small ball $B_{r}(q)$ such that $\bar{\Omega} \cap \overline{B_{r}(q)}$ is convex. Let us show that this implies $\bar{\Omega}$ is convex. Let $x$ and $y$ be arbitrary points in $\bar{\Omega}$. Choose some $p \in \bar{\Omega}$ and $R$ large enough so that $R>\max \{d(p, x), d(p, y)\}$. Then by the continuity argument there exists some $r_{0}$ such that $\overline{B_{r_{0}}(q)} \cap \bar{\Omega}$ is convex for any $q \in \bar{\Omega} \cap \overline{B_{R}(p)}$. Let $F$ be the set of piecewise smooth curves joining $x$ and $y$ lying entirely in $\bar{\Omega} \cap \overline{B_{R}(p)}$. Let $l$ be the infimum of the length of all such curves. Let $\left\{\gamma_{n}:[0, l] \rightarrow M\right\}$ be a sequence of curves in $F$ such that $\lim _{n \rightarrow \infty} L\left(\gamma_{n}\right)=l$. Take a partition of $[0, l], 0=t_{0}<t_{1}<\cdots<t_{k}=l$ such that $d\left(\gamma_{n}\left(t_{i}\right), \gamma_{n}\left(t_{i+1}\right)\right)<r_{0} / 2$ for all large $n$ and all $i=0,1, \ldots, k$. Replace each $\left.\gamma_{n}\right|_{\left[t_{i}, t_{i+1}\right]}$ with the geodesic segment joining $\gamma_{n}\left(t_{i}\right)$ and $\gamma_{n}\left(t_{i+1}\right)$, then it lies still in $\bar{\Omega}$ $\cap \overline{B_{R}(p)}$ by the local convexity. Let us still call these broken geodesics $\left\{\gamma_{n}\right\}$. It is clear that $\lim _{n \rightarrow \infty} L\left(\gamma_{n}\right)=l$. Since $\bar{\Omega} \cap \overline{B_{R}(p)}$ is compact, we can extract a subsequence of $\left\{\gamma_{n}\right\}$, which we still call $\left\{\gamma_{n}\right\}$, such that $\gamma_{n}\left(t_{i}\right) \rightarrow q_{i} \in \bar{\Omega} \cap \overline{B_{R}(p)}$ for all $i=1,2, \ldots, k-1$. Then $\gamma_{n}$ converges to the broken geodesic $\gamma_{0}$ joining $x$ and $y$ with possible breaks $q_{1}, \ldots, q_{k-1}$. If some $q_{i}$ is a break, then choose points $a_{1}=\gamma_{n}\left(t_{i}-\varepsilon\right)$ and $a_{2}=\gamma_{n}\left(t_{i}+\varepsilon\right)$ for sufficiently small $\varepsilon$. Then we can shorten $\gamma_{0}$ by replacing $\left.\gamma_{1}\right|_{\left[t_{i}-\varepsilon, t_{i}+\varepsilon\right]}$ with the geodesic segment joining $a_{1}$ and $a_{2}$, which clearly lies in $\bar{\Omega}$ by the local convexity. This is a contradiction. Thus $\gamma_{0}$ is a smooth geodesic segment. Since $M$ is a Cartan-Hadamard manifold, geodesics are unique. Thus the proof is complete. 
TheOREM 4.2. Let $M, \Omega, S$ and $N$ be as in Theorem 4.1. Define the positive normal bundle $N^{+} S=\{a N \mid a>0\}$ of $S$. Then exp: $N^{+} S \rightarrow M-\bar{\Omega}$ is a diffeomorphism.

Proof. Notice that exp: $N^{+} S \rightarrow M-\bar{\Omega}$ is 1-1. If not, then there exist $q \in M-\bar{\Omega}$ and $x, y \in S, x \neq y$, such that the geodesics $\gamma_{q x}$ and $\gamma_{q y}$ meet with $S$ perpendicularly. Since $\bar{\Omega}$ is convex $\gamma_{x y}$ lies in $\bar{\Omega}$, thus the geodesic triangle formed by $x, y$ and $q$ has the interior angle sum $>\pi$, which is a contradiction. It remains to show that there are no focal points of $S$. Suppose not, then there exist $q \in M-\bar{\Omega}$ and $p \in S$ and a Jacobi field $J$ along $\gamma_{p q}$ such that $J$ is perpendicular to $\gamma_{p q}^{\prime}(t)$ and $J=0$ at $q$. Let $l=d(p, q)$. Then by the usual argument which shows that geodesics stop minimizing beyond first conjugate point, we can obtain a geodesic from some point $x \in S$ to $\gamma_{p q}(l+\varepsilon)$ of length $<l+\varepsilon$ for sufficiently small $\varepsilon>0$. But obviously $d\left(S, \gamma_{p q}(l+\varepsilon)\right)=l+\varepsilon$. Thus the proof is complete.

THEOREM 4.3. Let $M$ be an $n$-dimensional Cartan-Hadamard manifold with the sectional curvature $\leqslant-1$. Let $\Omega, S$ and $N$ be the same as in Theorem 4.1. By Theorem 4.2 we can define the distance function s from $S$ on $M-\bar{\Omega}$. If $S$ is $C^{\infty}$, then $s$ is also $C^{\infty}$, and $\Delta s \geqslant(n-1) \tanh s$.

Proof. It is easy to see that $s$ is $C^{\infty}$ if $S$ is $C^{\infty}$ by using Theorem 4.2. Let $q$ be a point in $M-\bar{D}$, and let $b=d(q, S)$. Let $p$ be a point in $S$ with $d(p, q)=b$. Also let $\gamma:[0, b] \rightarrow M$ be the unit speed geodesic such that $\gamma(0)=p$ and $\gamma(b)=q$. Notice that $\gamma^{\prime}(0)$ is perpendicular to $S$ at $p$. Let $X_{1}(t), \ldots, X_{n}(t)$ be an O.N. frame parallel along $\gamma$, and $X_{n}(t)=T(t)=\gamma^{\prime}(t)$. Let $c$ be the geodesic starting from $q$ with $c^{\prime}(0)=X_{1}(b)$. Define $\gamma_{w}$ to be the geodesic that realizes the distance from $c(w)$ to $S$. Let $W(t)$ be the transversal Jacobi field along $\gamma$ obtained by varying this one parameter family of geodesics. Clearly $\langle W, T\rangle \equiv 0$ along $\gamma$ and $W(0)$ is tangent to $S$, and $W(b)=X_{1}(b)$. By the second variation formula

$$
\begin{aligned}
\frac{d^{2}}{d w^{2}} L\left(\gamma_{w}\right) & =\left.\left\langle D_{W} W, T\right\rangle\right|_{0} ^{b}+\int_{0}^{b}|\dot{W}|^{2}-\langle R(T, W) T, W\rangle d t \\
& =-\left\langle B_{W W}, T\right\rangle_{\text {at } p}+\int_{0}^{b}|\dot{W}|^{2}-\langle R(T, W) T, W\rangle d t \\
& \geqslant \int_{0}^{b}|\dot{W}|^{2}-\langle R(T, W) T, W\rangle d t
\end{aligned}
$$

Let $H^{n}$ be the simply connected space form of sectional curvature $\equiv-1$. Choose a unit speed geodesic $\tilde{\gamma}$ in $H^{n}$ of length $b$, and let $E_{1}(t), \ldots, E_{n}(t)$ be an O.N. frame parallel along $\tilde{\gamma}$, and $\tilde{\gamma}^{\prime}(t)=E_{n}(t)$. In $M$, let $W(t)=\sum_{i=1}^{n-1} h_{i}(t) X_{i}(t)$. Define $\tilde{W}(t)=\sum_{i=1}^{n-1} h_{i}(t) E_{i}(t)$. Let $J$ be a Jacobi field in $H^{n}$ along $\tilde{\gamma}$ such that $J(0)=$ $\tilde{W}(0)$ and $J(b)=\tilde{W}(b)$. Clearly $|W(t)|=|\tilde{W}(t)|$ and $|\dot{W}(t)|=\left|\tilde{W}^{\prime}(t)\right|$; thus $\langle R(T, W) T, W\rangle \leqslant\left\langle R\left(E_{n}, \tilde{W}\right) E_{n}, \tilde{W}\right\rangle$ where $R$ is the curvature tensor of $M$ or $H^{n}$ understood in appropriate context. We get

$$
\begin{aligned}
\int_{0}^{b}|\dot{W}|^{2}-\langle R(T, W) T, W\rangle d t & \geqslant \int_{0}^{b}\left|\tilde{W}^{\prime}\right|^{2}-\left\langle R\left(E_{n}, \tilde{W}\right) E_{n}, \tilde{W}\right\rangle d t \\
& \geqslant \int_{0}^{b}\left|J^{\prime}\right|^{2}-\left\langle R\left(E_{n}, J\right) E_{n}, J\right\rangle d t
\end{aligned}
$$


The last inequality is true because of the minimizing property of Jacobi field [GKM, p. 145]. Because of the symmetry of $H^{n}, J=\sum_{i=1}^{n-1} J_{i}$ where $J_{i}$ is a Jacobi field of the form $J_{i}(t)=f_{i}(t) E_{i}(t)$ with $f_{i}(0)=h_{i}(0)$ and $f_{i}(b)=h_{i}(b)$. In particular $h_{1}(b)=$ $f_{1}(b)=1$, and $h_{i}(b)=0$, for $2 \leqslant i \leqslant n-1$. For convenience define the index form $I(X, Y)$ for the vector fields $X, Y$ along $\tilde{\gamma}$ such that $X, Y \perp \tilde{\gamma}^{\prime}$ by

$$
I(X, Y)=\int_{0}^{b}\left\langle X^{\prime}, Y^{\prime}\right\rangle-\left\langle R\left(E_{n}, X\right) E_{n}, Y\right\rangle d t .
$$

Then

$$
I(J, J)=\sum_{i, j=1}^{n} \int_{0}^{b}\left\langle J_{i}^{\prime}, J_{j}^{\prime}\right\rangle-\left\langle R\left(E_{n}, J_{i}\right) E_{n}, J_{j}\right\rangle d t .
$$

But $J_{i}^{\prime}=f_{i}^{\prime} E_{i}$ and $\left\langle R\left(E_{n}, J_{i}\right) E_{n}, J_{j}\right\rangle=f_{i} f_{j} R_{n i n j}=0$ for $i \neq j$. Thus we obtain

$$
\begin{aligned}
I(J, J) & =\sum_{i=1}^{n-1} I\left(J_{i}, J_{i}\right) \geqslant I\left(J_{1}, J_{1}\right) \\
& \geqslant \tanh b, \quad \text { by the following Lemma } 4.4, \\
& =\tanh s, \quad \text { since } s=b \text { at } q .
\end{aligned}
$$

Repeating this argument w.r.t. $X_{2}, \ldots, X_{n-1}$, we obtain $\Delta s \geqslant(n-1) \tan s$, since the second derivative of $s$ in the direction of $X_{n}$ is zero.

LEMMA 4.4. Let $\gamma$ be a geodesic in $H^{n}$ with length $b$. Suppose $J$ is a Jacobi field along $\gamma$ with $|J(b)|=1$ and $\langle J, \dot{\gamma}\rangle \equiv 0$ and $J=f E$ for some function $f:[0, b] \rightarrow R$, where $E$ is a parallel vector field along $\gamma$. Let $T=\dot{\gamma}$. Then

$$
\int_{0}^{b}|\dot{J}|^{2}-\langle R(T, J) T, J\rangle d t \geqslant \tanh b
$$

Proof. $f$ satisfies the equation $f^{\prime \prime}-f=0$ with the condition $f(b)=1$ and $f(0)=u$ for some $u$. Thus $f(t)=c_{1} \cosh t+c_{2} \sinh t$ where $c_{1}=u$ and $c_{2}=$ $(1-u \cosh b) / \sinh b$.

Now

$$
\int_{0}^{b}|\dot{J}|^{2}-\langle R(T, J) T, J\rangle d t=\int_{0}^{b}\left|f^{\prime}\right|^{2}+|f|^{2} d t .
$$

Computing $f^{\prime}$ and $f$, we have

$$
\left|f^{\prime}\right|^{2}+|f|^{2}=\left[c_{1}^{2}+c_{2}^{2}\right] \cosh 2 t+2 c_{1} c_{2} \sinh 2 t
$$

So

$$
\int_{0}^{b}\left|f^{\prime}\right|^{2}+|f|^{2} d t=\left[c_{1}^{2}+c_{2}^{2}\right] \frac{\sinh 2 b}{2}+2 c_{1} c_{2}(\sinh b)^{2}
$$

Now

$$
\begin{aligned}
c_{1}^{2}+c_{2}^{2} & =u^{2}\left[1+\left(\frac{\cosh b}{\sinh b}\right)^{2}\right]+u\left[\frac{-2 \cosh b}{(\sinh b)^{2}}\right]+\frac{1}{(\sinh b)^{2}}, \\
c_{1} c_{2} & =u^{2}\left[\frac{-\cosh b}{\sinh b}\right]+u \frac{1}{\sinh b}
\end{aligned}
$$


Thus

$$
\begin{aligned}
\int_{0}^{b}\left|f^{\prime}\right|^{2}+|f|^{2} d t & =\frac{\cosh b}{\sinh b} u^{2}-\frac{2}{\sinh b} u+\frac{\cosh b}{\sinh b} \\
& =\frac{\cosh b}{\sinh b}\left[\left(u-\frac{1}{\cosh b}\right)^{2}-\frac{1}{(\cosh b)^{2}}+1\right] \\
& \geqslant \tanh b, \text { for any } u .
\end{aligned}
$$

Hence this completes the proof since the above inequality holds for any initial value $u=f(0)$.

We will first prove that the asymptotic Dirichlet problem is solvable if $M$ is a Cartan-Hadamard manifold of dimension 2. Even though this theorem is a consequence of Theorem 4.7, we will present Theorem 4.5 first for the clarity of exposition. Also this kind of barrier construction is the key idea of $\S 5$.

TheOREM 4.5. Let $M$ be a two-dimensional Cartan-Hadamard manifold with sectional curvature $K_{M} \leqslant-c^{2}$, for some $c>0$. Then the asymptotic Dirichlet problem is solvable.

Proof. By multiplying the metric tensor with a suitable constant, we may assume $K_{M} \leqslant-1$. Fix a pole $p \in M$. As mentioned earlier we have only to construct a barrier at any $v \in S_{p}$ with any sufficiently small angle $\delta>0$. Thus let us fix $v \in S_{p}$ and small $\delta, 0<\delta<\pi / 4$. Let $w_{1}$ and $w_{2} \in S_{p}$ be such that $\Varangle_{p}\left(v, w_{1}\right)=\Varangle_{p}\left(v, w_{2}\right)$ $=\delta$. Notice any maximal geodesic $\gamma$ of $M$ divides $M$ into two components each of which is convex. Let $D$ be one side of $\gamma$, and let $s$ be a function defined on $D$ which is the distance function from $\gamma$. We claim $\Delta \tanh s / 2 \geqslant 0$, on $D$. For,

$$
\begin{aligned}
\Delta \tanh \frac{s}{2} & =\frac{1}{2} \frac{1}{(\cosh s / 2)^{2}} \Delta s-\frac{1}{2} \frac{\sinh s / 2}{(\cosh s / 2)^{3}} \\
& \geqslant \frac{1}{2(\cosh s / 2)^{2}}\left[\tanh s-\tanh \frac{s}{2}\right], \quad \text { by Theorem 4.3, } \\
& \geqslant 0 .
\end{aligned}
$$

Let $\Omega$ be the sector formed by two geodesic rays $\gamma_{w_{1}}$ and $\gamma_{w_{2}}$. Let $s_{1}$ be the distance function from $\gamma_{w_{1}}$ defined on $\Omega$. Similarly define $s_{2}$ to be the distance function from $\gamma_{w_{2}}$ defined also on $\Omega$. We showed that $\beta_{1}=\tanh \left(s_{1} / 2\right)+\tanh \left(s_{2} / 2\right)-2$ is subharmonic on $\Omega$. Notice that $\beta_{1} \rightarrow 2$ as $q \rightarrow \gamma_{w}(\infty)$ for any $w \in S_{p}$ such that $\Varangle_{p}(v, w)<\delta$, since $s_{i} \rightarrow \infty$ along any ray $\gamma_{w}$ with $\Varangle_{p}(v, w)<\delta$. Define $\Omega_{1}$ and $\Omega_{2}$ as follows.

$$
\begin{aligned}
& \Omega_{1}=\left\{q \in \Omega \mid d(p, q)>1, s_{1}(q)>1 \text { and } s_{2}(q) \geqslant 1\right\}, \\
& \Omega_{2}=\left\{q \in \Omega \mid d(p, q) \geqslant 2, s_{1}(q) \geqslant 2 \text { and } s_{2}(q) \geqslant 2\right\} .
\end{aligned}
$$

Let $\Omega_{3}=\Omega_{1}-\Omega_{2}$. Let $\eta=\sup \left\{\beta_{1}(q) \mid q \in \Omega_{3}\right\}$. Clearly $q \in \Omega_{1}$, then it is clear that $\beta \equiv \eta$ on the open set $\Omega_{3}$. Thus we can extend $\beta$ to the whole $M-\Omega_{2}$ as a constant function. Then $\beta$ is the desired barrier at $v$ with angle $\delta$. Since $v$ and $\delta$ are arbitrary, we can solve the asymptotic Dirichlet problem by Theorem 2.7 . 
To generalize this construction to the higher dimension we need the following definition.

Definition 4.6. Let $M$ be a Cartan-Hadamard manifold. We say $M$ satisfies the convex conic neighborhood condition at $x \in M(\infty)$ if for any $y \in M(\infty), y \neq x$, there exist $V_{x}$ and $V_{y} \subset \bar{M}$ such that $V_{x}$ and $V_{y}$ are disjoint open sets of $\bar{M}$ in terms of the cone topology and $V_{x} \cap M$ is convex with $C^{2}$ boundary. If this condition is satisfied for all $x \in M(\infty)$, we say $M$ satisfies the convex conic neighborhood condition.

THEOREM 4.7. Let $M$ be an $n$-dimensional Cartan-Hadamard manifold with sectional curvature $\leqslant-c^{2}$, for some constant $c>0$. Suppose, furthermore, $M$ satisfies the convex conic neighborhood condition. Then the asymptotic Dirichlet problem is solvable.

Proof. As usual we could assume $c=1$. To prove the theorem we have only to construct a barrier at $v$ with angle $\delta$ for any $v \in S_{p}$ and small $\delta>0$. In what follows $p, v \in S_{p}$ and $\delta>0$ are arbitrarily chosen, but fixed. Define $S(v, \delta)=\left\{w \in S_{p} \mid\right.$ $\left.\Varangle_{p}(v, w)=\delta\right\}$. For each $w \in S(v, \delta)$, apply the convex conic neighborhood condition to $\gamma_{w}(\infty)$ and $\gamma_{v}(\infty)$ to obtain disjoint $V_{w}$ and $O_{w}$ such that $V_{w}$ is an open neighborhood of $\gamma_{w}(\infty)$ and $O_{w}$ an open neighborhood of $\gamma_{v}(\infty)$ in the cone topology, and $V_{w} \cap M$ is convex with $C^{2}$ boundary. Let $U_{w}=\left\{z \in S_{p} \mid \gamma_{z}(\infty) \in V_{w}\right\}$, then the set $\left\{U_{w}\right\}_{w \in S(v, \delta)}$ is an open covering of the compact set $S(v, \delta) \subset S_{p}$. Thus there exist finite $w_{1}, \ldots, w_{k} \in S(v, \delta)$ such that $\left\{U_{w_{i}}\right\}$ covers $S(v, \delta)$. We could also assume none of $V_{w_{i}}$ contains any $V_{w_{j}}$ for $i \neq j$. Let

$$
\Omega=M-\bigcup_{i=1}^{k} V_{w_{i}}=\bigcap_{i=1}^{k}\left(M-V_{w_{i}}\right) \text {. }
$$

Notice that the distance function $s_{i}$ defined on $M-V_{w_{i}}$ from $\partial\left(M-V_{w_{i}}\right)$ is $C^{2}$ and it is easy to check $\Delta \tanh s_{i} / 2 \geqslant 0$ using Theorems 4.2 and 4.3 as before. The function

$$
\beta_{1}=\sum_{i=1}^{k} \tanh \frac{s_{i}}{2}-k
$$

is defined on $\Omega$ and is subharmonic. Let $V_{v}=\cap_{i=1}^{k} O_{w_{i}}$. It is clear $\beta_{1} \rightarrow 0$ as $q \rightarrow x$, for any $x \in V_{v} \cap M(\infty)$. Since each $V_{w_{i}}$ contains a truncated cone, we can take sufficiently large $R$ such that, for any $q \in \Omega$ and $d(p, q) \geqslant R, \Varangle_{p}\left(\gamma_{p q}^{\prime}(0), v\right)<\delta$. Define $\Omega_{1}$ and $\Omega_{2}$.

$$
\begin{aligned}
& \Omega_{1}=\left\{q \in \Omega \mid d(p, q) \geqslant 2 R \text { and } s_{i}(q) \geqslant 2, \text { for } i, \ldots, k\right\}, \\
& \Omega_{2}=\left\{q \in \Omega \mid d(p, q)>R \text { and } s_{i}(q)>1, \text { for } i=1, \ldots, k\right\} .
\end{aligned}
$$

Then $\Omega_{3}=\Omega_{1}-\Omega_{2}$ is an open set of $M$ and the proof now proceeds exactly as in the proof of Theorem 4.5 .

REMARK 4.8. If $n=2$, then $M$ satisfies the convex conic neighborhood condition automatically, thus Theorem 4.5 follows from Theorem 4.7. If $M$ is rotationally symmetric, then by Theorem 3.7 it also satisfies the convex cone neighborhood condition. Thus Theorem 4.7 can be regarded as a generalization of Theorem 3.6, even though the curvature assumption here is stronger. 
REMARK 4.9. Recently D. Sullivan [S] showed that the asymptotic Dirichlet problem is solvable if the sectional curvature is pinched between two negative constants. His method is based on the probability theory. M. Anderson [An] proved that the convex conic neighborhood condition is satisfied if the sectional curvature is pinched between two negative constants.

5. Extension to nonsimply connected manifolds. In this section we will extend the asymptotic Dirichlet problem to certain classes of nonsimply connnected manifolds. We will be, however, mostly concerned with surfaces, because the special property of dimension 2 makes it possible to construct barriers by naturally adopting the ideas of $\S 4$, even though the problem could be formulated just as easily in higher dimensions.

Throughout this section, $M$ denotes a two-dimensional complete Riemannian manifold. (In short, a complete surface.) We denote the Gaussian curvature function by $K_{M} . M$ is assumed to be noncompact and finitely connected which means by definition that the fundamental group of $M$ is finitely generated. Topologically, $M$ is a compact surface with finitely many points removed. Since $M$ is complete, a sufficiently small neighborhood of each puncture could be visualized as an infinite tube, which we call an end. More precisely, we introduce

Definition 5.1. An end of $M$ is a function $\varepsilon$ that assigns to each compact subset $K$ of $M$ a connected component $\varepsilon(K)$ of $M-K$ such that $\varepsilon(K) \supset \varepsilon(L)$ if $K \subset L$. A curve $\sigma:[0, \infty) \rightarrow M$ is said to converge to an end $\varepsilon$ if for any compact $K \subset M, \varepsilon(K)$ contains a terminal segment of $\sigma$.

Let $H$ be the universal covering manifold of $M$, then it is well known that $M$ is isometric to $H / D$ for some subgroup $D$ of the isometry group of $H$ such that $D$ is isomorphic to $\pi_{1}(M)$ and $D$ acts on $H$ properly discontinuously with no fixed points. We will describe the geometry of ends following Eberlein [E1 and E2]. It is easy to see that every isometry $\phi$ of $H$ extends to $H$ as a homeomorphism w.r.t. the cone topology. Let $D$ be as above.

Definition 5.2. The limit set $L(D)$ is the set of accumulation points in $H(\infty)$ of an orbit $D(p)$ of some point $p \in H$. Eberlein [E1, p. 12] showed that $L(D)$ does not depend on the choice of $p$. The set of ordinary points is defined to be $O(D)=H(\infty)$ $-L(D)$.

Definition 5.3. An isometry $\phi$ of $H$ is called parabolic if it has exactly one fixed point in $H(\infty)$. A geodesic $\gamma$ in $M$ is called parabolic if for some lift $\tilde{\gamma}$ of $\gamma$ to $H$ the point $\tilde{\gamma}(\infty) \in H(\infty)$ is fixed by a parabolic isometry in $D$. A geodesic $\gamma$ is said to be expanding if for any lift $\tilde{\gamma}$ of $\gamma$ to $H, \tilde{\gamma}(\infty) \in O(D)$. An end $\varepsilon$ is called a parabolic end (resp. expanding end) if there exists a parabolic (resp. expanding) geodesic converging to $\varepsilon$.

The following theorem is taken from Eberlein [E1, pp. 56 and 92].

THEOREM 5.4. Let $M=H / D$ be a noncompact, finitely connected, complete surface with $K_{M} \leqslant-c^{2}<0$. Then:

(1) All ends are either expanding or parabolic.

(2) A parabolic end has a neighborhood with finite total curvature. 
(3) Assume that $|L(D)| \geqslant 2$ and $M$ is not topologically a Moebius band, then each expanding end has a closed neighborhood $U$ with geodesic coordinates $s, \theta$ where $s \geqslant 0$ and $\theta \in \mathbf{R} / 2 \pi$ so that the metric is of the form $d s^{2}+g(s, \theta)^{2} d \theta^{2}$ where $g$ is a $C^{\infty}$ function. The s parameter curve $\gamma_{\theta}:[0, \infty) \rightarrow M$ with fixed $\theta$ is a minimizing geodesic ray, and the curve $c_{0}: \theta \mapsto(0, \theta)$ is a simple closed periodic geodesic, which we call the separating geodesic of $U$. Let $A_{\theta_{1}}$ be the geodesic distance function from the ray $\gamma_{\theta_{1}}$, then $\lim _{s \rightarrow 0} A_{\theta_{1}}\left(\gamma_{\theta_{2}}(s)\right)=\infty$ for $\theta_{1} \neq \theta_{2} \bmod 2 \pi$.

In fact, part (3) of Theorem 5.4 is not surprising. We will give a heuristic argument here. To avoid technicalities let us assume that $M$ is topologically a nonsimply connected compact surface minus one puncture. Take a closed curve $C$ that cuts out a neighborhood of the puncture. Suppose that the end corresponding to the puncture is "expanding," then obviously the infimum of the arc lengths of all curves freely homotopic to $C$ must be greater than zero. By a standard argument we can take a curve $\gamma$ in the same free homotopy class as $C$ such that $\gamma$ realizes the infimum length, so $\gamma$ must be a closed geodesic. Then cut out a neighborhood of the puncture with $\gamma$, and call this neighborhood $U . U$ is a complete surface with compact totally geodesic boundary $\gamma$. Since $K_{M} \leqslant-c^{2}<0$, it is not hard to see that exp: $V^{+} \rightarrow U$ is a diffeomorphism where $V^{+}$is the set of vectors of the normal bundle of $\gamma$ pointing toward $U$. All proofs should follow easily from the standard Jacobi field arguments.

The above theorem shows that an expanding end behaves as its name suggests. Parabolic ends are in a sense shrinking. The following version due to Finn [F, Theorem 17] is most suitable for our purpose.

THEOREM 5.5. Let $M$ be a complete surface with an end $\varepsilon$. Let $U$ be a neighborhood of $\varepsilon$ such that $K_{M} \leqslant 0$ in $U$ and the total curvature in $U$ is finite. Then the complex structure of $U$ that comes from isothermal coordinates is equivalent to that of the punctured unit disk in $\mathbf{C}$.

Definition 5.6. Let $M$ be a complete surface. An end $\varepsilon$ is called a standard expanding end if $\varepsilon$ has a neighborhood $U$ such that $K_{M} \leqslant-c^{2}$ in $U$ for some $c>0$, and the conditions on $U$ in part (3) of Theorem 5.4 are satisfied. Thus in our notation the standard expanding end denotes the specific neighborhood $U$ which is cut out by the separating geodesic. An end $\varepsilon$ is called a standard shrinking end if $\varepsilon$ has a neighborhood $U$ such that the complex structure of $U$ that comes from isothermal coordinates is equivalent to that of the punctured unit disk in $\mathbf{C}$. Let $u$ be a (sub)harmonic function defined on a neighborhood $U$ of a standard shrinking end $\varepsilon$. We say $u$ extends to the puncture of $\varepsilon$ as a (sub)harmonic function if there exists a (sub)harmonic function $v$ defined on the unit disk $D$ such that $v \equiv u$ on $D-\{0\}$ whereas $U$ is biholomorphically identified with $D-\{0\}$.

There are numerous examples of complete surfaces with finitely many standard ends. One way of producing such an example is by attaching ends [E2]: Let $[-2, \infty) \times S^{1}$ be the Riemannian manifold with coordinates $s \geqslant-2$ and $\theta \in \mathbf{R} / 2 \pi$ with metric $d s^{2}+(\cosh s)^{2} d \theta^{2}$. Then $[0, \infty) \times S^{1}$ is obviously a standard expanding end. Let $M$ be any complete surface. Take away a small disk from $M$ and attach $[-2, \infty) \times S^{1}$ to the hole. One can then define a new Riemannian metric on $M$ with 
the above end attached without changing the Riemannian metric on $[-1, \infty) \times S^{1}$ by the usual partition of unity argument. One can similarly attach a standard shrinking end. Our Dirichlet problem is defined on standard expanding ends, whereas we have to exercise a little bit of caution on standard shrinking ends.

DEFINITION 5.7. Let $U$ be a standard expanding end which is diffeomorphic to $[0, \infty) \times S^{1}$. We will use the notations in Theorem 5.4. Let $\delta>0$ and $r>0$. Fix a point $p$ on the separating geodesic of $U$. Define the truncated sector $K(p, \delta, r)=\{q$ $\in U \| \theta(q)-\theta(p) \mid<\delta$ and $s(q)>r\}$. Throughout this section $\gamma_{p}$ denotes the $s$ parameter geodesic ray from $p$. Thus $\gamma_{p}$ meets with the separating geodesic perpendicularly. Let $u$ be a real valued function defined on $U$. We say $u$ converges to a number $A$ as $q \rightarrow \gamma_{p}(\infty)$, if given $\varepsilon>0$, there exist some $\delta>0$ and $r>0$ such that $|u(q)-A|<\varepsilon$ for all $q \in K(p, \delta, r)$. We define limsup and liminf similarly in terms of truncated sectors.

Definition 5.8. Let $M$ be a finitely connected, complete surface with $k$ standard expanding ends, $k \geqslant 1$. Let $C_{1}, \ldots, C_{k}$ be the separating geodesics of those ends. We also assume that all ends are either standard expanding or standard shrinking. Let $\phi_{i}: C_{i} \rightarrow \mathbf{R}$ be continuous functions for all $i=1, \ldots, k$. Then the asymptotic Dirichlet problem on standard expanding ends is to find a harmonic function $u$ on $M$ such that $u$ extends to the puncture of every standard shrinking end and for any $C_{i}$ and any point $p \in C_{i}$

$$
\lim _{q \rightarrow \gamma_{p}(\infty)} u(q)=\phi_{i}(p)
$$

in the sense of Definition 5.7.

The proof of the solution of the above problem is very similar to that given before. Most crucial is the following version of asymptotic maximum principle.

Proposition 5.9. We will use the same, notation as in Definition 5.8.

(1) Suppose $f: M \rightarrow \mathbf{R}$ is a subharmonic function such that $u$ extends to the puncture of every standard shrinking end as a subharmonic function and $\lim \sup f(q) \leqslant 0$ as $q \rightarrow \gamma_{p}(\infty)$ for any $C_{i}$ and any point $p \in C_{i}$. Then $f \leqslant 0$ on $M$.

(2) Let $f$ (resp. $g$ ) be a subharmonic (resp. superharmonic) function on $M$ which extends to the puncture of every standard shrinking end as a subharmonic (resp. superharmonic) function. Suppose that for any $C_{i}$ and any $p \in C_{i}$

$$
\limsup _{q \rightarrow \gamma_{p}(\infty)} f(q) \leqslant \liminf _{q \rightarrow \gamma_{p}(\infty)} g(q) \text {. }
$$

Then $f \leqslant g$ on $M$.

Proof. Let $A$ be the supremum of $f$ on $M$. Assume $A>0$. Using the same idea as in the proof of Proposition 2.5 we can find closed curves $\tilde{C}_{1}, \ldots, \tilde{C}_{k}$ which cut out neighborhoods $\tilde{V}_{i}$ of standard expanding ends such that $f \leqslant A_{1}<A$ in each $\tilde{V}_{i}$. Let $\tilde{M}=M-\cup_{i=1}^{k} \tilde{V}_{i}$. Then $\tilde{M}$ is a complete manifold with compact boundaries. Suppose there exists a sequence $\left\{q_{i}\right\} \subset M$ such that $f\left(q_{i}\right) \rightarrow A$ as $i \rightarrow \infty$ and $q_{i}$ converges to some standard shrinking end $\varepsilon$. Take a neighborhood $U$ of $\varepsilon$ which is biholomorphic to the punctured unit disk $D-\{0\}$. We can then consider $u$ as a 
subharmonic function defined on $D$. The above assumption says that $u(0)=A$. Thus by the usual maximum principle $U \equiv A$ on $D$. Cut away a neighborhood of $\varepsilon$ from $\tilde{M}$ by the closed curve which corresponds to the circle $|z|=\frac{1}{2}$ under the biholomorphism of $U$ to $D-\{0\}$. Also cut away a neighborhood of every other standard shrinking end from $\tilde{M}$. Thus we obtain a domain $D$ of $M$ with compact closure. It is clear that $f$ has an interior maximum in $D$, thus $f \equiv A$ on $D$. But $f \leqslant A_{1}<A$ on each $\tilde{C}_{i}$. This is a contradiction. Thus we can assume that there exists some $B<A$ such that each standard shrinking end has a neighborhood on which $f \leqslant B$. Then again cut away all these neighborhoods from $\tilde{M}$ to obtain a domain $\tilde{D}$ of $M$ with compact closure. Clearly $f$ attains a maximum $A$ at an interior point of $\tilde{D}$, and $f \leqslant$ $\max \left(B, A_{1}\right)<A$ on $\partial \tilde{D}$, which is again a contradiction. Thus it is easy to conclude that $A \leqslant 0$. Applying (1) to the subharmonic function $f-g$, we obtain (2) immediately.

THEOREM 5.10. Let $M$ be a finitely connected, complete surface with $k$ standard expanding ends, $k \geqslant 1$. Assume also that all ends are either standard expanding or standard shrinking. Then the asymptotic Dirichlet problem posed in Definition 5.8 is solvable, hence $M$ possesses many nonconstant bounded harmonic functions.

Proof. The proof is very similar to what was done before, but there are some subtle points because of the presence of the shrinking ends. First define $F=\{f: M$ $\rightarrow \mathbf{R} \mid f$ is a subharmonic function which extends to the puncture of every standard shrinking end as a subharmonic function, and $\lim \sup f(q) \leqslant \phi_{i}(p)$ as $q \rightarrow \gamma_{p}(\infty)$ for any $C_{i}$ and any $p \in C_{i}$. Notice that $F$ is nonempty, because $F$ contains functions identical to sufficiently negative constants. Let $u(q)=\sup \{f(q) \mid$ if $\in F\}$, then $u: M \rightarrow \mathbf{R}$ is obviously harmonic. To prove the convergence to the asymptotic boundary values we need barriers defined as follows. Fix some $C_{i}$ and a point $p \in C_{i}$. A function $B: M \rightarrow \mathbf{R}$ is called a barrier at $p$ with angle $\delta$, if $B$ satisfies the following conditions:

(1) $B$ is a subharmonic function on $M$ which extends to the puncture of every standard shrinking end as a subharmonic function.

(2) $B \leqslant 0$ and $\lim B(q)=0$ as $q \rightarrow \gamma_{p}(\infty)$.

(3) $\exists \eta>0$ such that $\lim \sup B(q) \leqslant-\eta$ as $q \rightarrow \gamma_{a}(\infty)$, if $a \in C_{j}, j \neq i$, or $a \in C_{i}$ and $|\theta(a)-\theta(p)|>\delta$.

In view of Proposition 5.9 it is rather easy to see that the following version of Theorem 2.7 is true: If, for any $C_{i}$, any $p \in C_{i}$ and any sufficiently small $\delta>0$, there exists a barrier at $p$ with angle $\delta$, then the asymptotic Dirichlet problem of Definition 5.8 is solvable. The proof of convergence to the asymptotic boundary values on the standard expanding end is exactly the same as in the proof of Proposition 5.9. It remains to check if $u$ extends to the puncture of every standard shrinking end as a harmonic function. But $u$ is bounded above by the maximum $m_{0}$ of $\phi_{i}$ on $C_{i}$ for all $i=1, \ldots, k$, since by Proposition 5.9 every $f \in F$ is bounded above by $m_{0} . u$ is obviously bounded below by the minimum of $m_{1}$ of $\phi_{i}$ on $C_{i}$ for all $i=1, \ldots, k$. Then by the removable singularity theorem for bounded harmonic functions (e.g., see [Ma, Corollary of Theorem 5.9]), $u$ extends to the puncture. 
All we have to do is to construct a barrier. This procedure is the same as that of Theorem 4.5. Fix some $C_{i}$ and $p \in C_{i}$. Also fix some sufficiently small $\delta>0$. Let $p_{1}$ and $p_{2}$ be the points of $C_{i}$ such that $\left|\theta\left(p_{i}\right)-\theta(p)\right|=\delta$ for $i=1,2$. Let $\gamma_{p_{1}}, \gamma_{p}$ and $\gamma_{p_{2}}$ be the usual $s$-parameter geodesic rays from $p_{1}$, and $p_{2}$ respectively. Let $\Omega$ be the sector bounded by $\gamma_{p_{1}}$ and $\gamma_{p_{2}}$ and the geodesic segment of $C_{i}$ from $p_{1}$ to $p_{2}$.

Define $\tau_{i}$ to be the geodesic distance function from $\gamma_{p_{i}}$ defined on $\Omega$. Then as in the proof of Theorem 4.5, $\beta=\tanh \tau_{1} / 2+\tanh \tau_{2} / 2-2$ is a subharmonic function on $\Omega$. Let $\Omega_{1}=\left\{q \in \Omega \mid s(q)>1\right.$ and $\tau_{i}(q)>1$ for $\left.i=1,2\right\}$ and $\Omega_{2}=\{q \in \Omega \mid s(q) \geqslant 2$ and $\tau_{i}(q) \geqslant 2$ for $\left.i=1,2\right\}$. Then the rest of the proof is exactly the same as in the proof of Theorem 4.5. Notice that $\beta$ extends outside $\Omega_{1}$ as a negative constant. Thus it is trivial to check all three conditions of barrier.

REMARK 5.11. In Definition 5.8 and the subsequent solution, we implicitly assumed that $C_{i}$ 's are all distinct. But there is one exception, namely, a cylinder with both ends expanding. For example $\mathbf{R} \times S^{1}$ with coordinates $s \in \mathbf{R}, \boldsymbol{\theta} \in \mathbf{R} / 2 \pi$. Suppose it has a Riemannian metric $d s^{2}+(\cosh s)^{2} d \theta^{2}$, then obviously both ends are expanding and the curve $c_{0}: \theta_{1} \rightarrow(0, \theta)$ is the separating geodesic of both ends. Thus assume $M$ is topologically a cylinder with both ends expanding and the geodesic $c_{0}$ is the separating geodesic of both ends. Define $S_{1}=\left\{v \in M_{q} \mid\right.$ $\left\langle v, c_{0}^{\prime}(t)\right\rangle=0$ where $c_{0}(t)=q$ and for $t>0, \gamma_{v}(t)$ has positive $s$ coordinates $\}$. Let $S_{2}=\left\{v \mid-v \in S_{1}\right\}$. The Dirichlet data should be given on both $S_{1}$ and $S_{2}$, so Definition 5.8 should be modified as follows: Let $\phi_{1}: S_{i} \rightarrow \mathbf{R}$ be continuous functions for $i=1,2$. Find a harmonic function $u: M \rightarrow \mathbf{R}$ such that $\lim u(q)=\phi_{i}(v)$ as $q \rightarrow \gamma_{v}(\infty)$ in the sense of Definition 5.7. Evidently Proposition 5.9 and Theorem 5.10 are valid in this setting.

At this point, it should be noted that $M$ is not assumed to be orientable. That is why we have to work with the complex structure only in a neighborhood of standard shrinking ends. In fact if $M$ is orientable, then $M$ can be regarded as a Riemann surface, thereby making it possible to genuinely compactify all standard shrinking ends. This point can be pushed further to treat nonorientable surfaces as suggested by Professor $\mathrm{H}$. Wu: Let $M$ be a nonorientable surface with at least one standard expanding end. Assume also that all ends are standard. Let $\pi: \tilde{M} \rightarrow M$ be a double cover, thus $\tilde{M}$ is an orientable surface and each end of $M$ corresponds to two ends in $\tilde{M}$. Let $\sigma: \tilde{M} \rightarrow \tilde{M}$ be an involutive deck transformation. Then the Dirichlet data on each standard expanding end give rise to the same data on corresponding ends in $\tilde{M}$. $\tilde{M}$ becomes a Riemann surface, and we compactify all standard shrinking ends to form a new Riemann surface $M^{*}$. Note that $\tilde{M}$ as a Riemann surface is $M^{*}$ with finitely many points removed. Then solve the asymptotic Dirichlet problem on each standard expanding end of $M^{*}$ to obtain a harmonic function $\tilde{u}$ on $M^{*}$. Let $u=\frac{1}{2}\left(\tilde{u}+\sigma^{*} \tilde{u}\right)$, then $u$ is again a harmonic function on $\tilde{M}$ which is invariant under the involution $\sigma$. Thus $u$ can be considered as a function defined on $M$. It is clear that $u$ takes the correct boundary values given standard expanding ends.

One important aspect of classification theory of Riemann surfaces is the dichotomy of Riemann surfaces into two classes: ones that admit a nonconstant bounded harmonic function, ones that do not. As is the usual case, there are many satisfactory geometric conditions that guarantee the nonexistence of nonconstant bounded 
harmonic functions, whereas the existence results in geometric terms are rare. For the purpose of comparison, we quote the theorem of Huber [H, Theorem 15].

THEOREM 5.12. Let $M$ be an open Riemann surface with complete Riemannian metric such that $\int_{M} K^{-}>-\infty$, where $K^{-}=\min \left(K_{M}, 0\right)$. Then every bounded harmonic function on $M$ is constant.

Our result that complements Huber's is the following.

THEOREM 5.13. Let $M$ be a finite connected, complete surface (not necessarily orientable) with $K_{m} \leqslant-c^{2}$, for some constant $c>0$. Assume that $M$ is not topologically a Moebius band. If $\int_{M} K_{M}=-\infty$, then $M$ admits infinitely many nonconstant bounded harmonic functions.

Proof. Since $\int_{M} K_{M}=-\infty, M$ is noncompact. Let $M=H / D$. If $|L(D)|>2$, then by Theorem 5.3 all expanding ends are standard expanding, and all parabolic ends are standard shrinking using (2) of Theorem 4.3 and Theorem 5.5. Thus all ends are either standard expanding or standard shrinking. Since the total curvature is $-\infty$, there must be at least one expanding end, thus the conditions of Theorem 5.10 are satisfied. Thus by choosing nontrivial Dirichlet data $\phi_{i}$, we can obtain uncountably many nonconstant bounded harmonic functions. It remains to take care of two cases, i.e., $|L(D)|=0$ or 1. Eberlein [E1, p. 27] describes them as follows: If $L(D)$ is empty, then $M=H$, i.e., Cartan-Hadamard manifold. If $|L(D)|=1$, then $M$ is topologically a cylinder with one parabolic end and one expanding end. In the case where $M=H$, we can certainly pose an asymptotic Dirichlet problem w.r.t. a point, and solve it. So let $M$ be topologically a cylinder with one expanding end and one shrinking end. $M$ has coordinates $s, \theta$ where $s$ parameter curves are minimizing geodesic which are mutually divergent as $s \rightarrow \infty$. We can take a neighborhood of the parabolic end, which is a totally convex set with boundary curve $c: \theta \rightarrow(0, \theta)[\mathbf{E} 1, \mathrm{p} .95]$. We can then pose an asymptotic Dirichlet problem on the expanding end using $c$ instead of separating geodesics. Proofs work exactly the same way as before.

Corollary 5.14. Let $M$ be the same as in Theorem 5.10. Assume also that $M$ is orientable, thus $M$ is a Riemann surface. Then $M$ possesses infinitely many bounded holomorphic functions.

Proof. Let $u$ be a nonconstant bounded harmonic function. Fix a point $p \in M$. Define $v: M \rightarrow \mathbf{R}$ to be $v(q)=\int_{p}^{q} * d u$, where the integral is taken over some path from $p$ to $q$. Thus $v$ is defined up to integral multiples of $2 \pi$. Define $h=\exp (u+i v)$, then $h$ is a well-defined holomorphic function, and is bounded since $|f|=e^{u}$.

\section{BIBLIOGRAPHY}

[Al] L. Ahlfors, Sur le type d' une surface de Riemann, C. R. Acad. Sci. Paris 201 (1935), 30-32.

[An] M. Anderson, The Dirichlet problem at infinity for manifolds of negative curvature, preprint.

[AS] L. Ahlfors and L. Sario, Riemann surfaces, Princeton Univ. Press, Princeton, N. J., 1960.

[Ax] S. Alexander, Locally convex hypersurfaces of negatively curved spaces, Proc. Amer. Math. Soc. 64 (1977), 321-325.

[B] R. Bishop, Infinitesimal convexity implies local convexity, Indiana Univ. Math. J. 24 (1974), $169-172$. 
[Ch] S.-Y. Cheng, Liouville theorem for harmonic maps, Proc. Sympos. Pure Math., vol. 36, Amer. Math. Soc., Providence, R. I., 1980, pp. 147-151.

[Cil] H. I. Choi, On the Liouville theorem for harmonic maps, Proc. Amer. Math. Soc. 85 (1982), 91-94.

[Ci2] , Characterizations of simply connected rotationally symmetric manifolds, Trans. Amer. Math. Soc. 275 (1983), 723-727.

[Ci3] __ Thesis, Univ. of California, Berkeley, June 1982.

[El] P. Eberlein, Geodesics and ends in certain surfaces without conjugate points, Mem. Amer. Math. Soc. vol. 13, no. 199 (1978).

[E2] __ Surfaces of nonpositive curvature, Mem. Amer. Math. Soc. vol. 20, no. 218 (1979).

[EO] P. Eberlein and B. O'Neill, Visibility manifolds, Pacific J. Math. 46 (1973), 45-109.

[F] R. Finn, On a class of conformal metrics with applications to differential geometry in the large, Comment. Math. Helv. 40 (1965), 1-30.

[GT] D. Gilbarg and N. S. Trudinger, Elliptic partial differential equations of second order, SpringerVerlag, Berlin and New York, 1977.

[GWI] R. Greene and $\mathrm{H}$. Wu, Function theory on manifolds which possess a pole, Lecture Notes in Math., vol. 699, Springer-Verlag, Berlin and New York, 1979.

[GW2] __ On a new gap phenomenon in Riemannian geometry, Proc. Nat. Acad. Sci. U.S.A. (1982).

[GKM] D. Gromoll, W. Klingenberg and W. Meyer, Riemannsche Geometrie im Grossen, Lecture Notes in Math., vol. 55, Springer-Verlag, Berlin and New York, 1975.

[HJW] S. Hildebrandt, J. Jost and K.-O. Widman, Harmonic mappings and minimal submanifolds, Invent. Math. 62 (1980), 269-298.

[H] A. Huber, On subharmonic functions and differential geometry in the large, Comment. Math. Helv. 32 (1957), 13-72.

[Ma] A. Markushevich, Theory of functions of a complex variable, vol. 2, Chelsea, New York, 1967.

[Mi] J. Milnor, On deciding whether a surface is parabolic or hyperbolic, Amer. Math. Monthly 84 (1977), 43-46.

[S] D. Sullivan, The Dirichlet problem at infinity for a negatively curved manifold, preprint.

[W] H. Wu, Normal families of holomorphic mappings, Acta Math. 119 (1967), 193-233.

[Ya] P. Yang, Curvature of complex submanifolds of $\mathbf{C}^{n}$, J. Differential Geom. 12 (1977), 499-511.

[Yu] S.-T. Yau, Harmonic functions on complete Riemannian manifolds, Com. Pure Appl. Math. 28 (1975), 201-228.

Department of Mathematics, University of Chicago, Chicago, Illinois 60637 